\title{
Practical Dynamic Consensus of Stuart-Landau oscillators over Heterogeneous Networks
}

\author{
E. Panteley ${ }^{\mathrm{a}, \mathrm{b}}$, A. Loría ${ }^{\mathrm{a}}$, and A. El-Ati ${ }^{\mathrm{c}}$ \\ ${ }^{a}$ Laboratoire des signaux et systèmes, CNRS, Univ Paris Saclay, 91192 Gif-sur-Yvette, \\ France; \\ bITMO University, Kronverkskiy av. 49, Saint Petersburg, 197101, Russia; \\ ${ }^{\mathrm{c}}$ Institut Polytechnique des Sciences Avancées (IPSA), 63 Bis, Boulevard de Brandebourg, \\ 94200 Ivry-sur-Seine.
}

\author{
ARTICLE HISTORY \\ Compiled October 11, 2018
}

\begin{abstract}
In Panteley and Loria (2017) a framework for the study of synchronization and collective behaviour of networked heterogeneous systems was introduced. It was underlined that in such scenario an emergent collective behaviour arises, one that is inherent to the network and that is independent of the interconnection strength. Therefore, the natural way to make complete study of synchronization is by investigating, on one hand, the stability of the emergent dynamical system and, on the other, by assessing the difference between the motion of each individual system and that of the emergent one. Thus, if all systems' motions approach that of the emergent dynamics, we say that they reach dynamic consensus. In this paper we study dynamic consensus of a fairly general class of nonlinear heterogeneous oscillators, called Stuart-Landau. We establish that the emergent dynamics consists in that of an "averaged" oscillator with a global attractor that consists in a limit-cycle and, moreover, we determine its frequency of oscillation. Then, we show that the heterogeneous oscillators achieve practical dynamic consensus that is, their synchronization errors measured relative to the collective motion, are ultimately bounded.
\end{abstract}

\section{KEYWORDS}

Networked systems, consensus, nonlinear oscillators; robust stability; synchronization

\section{Introduction}

In its simplest form, consensus consists in a group of linear interconnected systems synchronizing their trajectories and asymptotically reaching a common equilibrium point determined by the systems' intial conditions (Olfati-Saber \& Murray, 2004; Ren et al., 2007). Furthermore, when the network is heterogeneous, that is, when it comprises systems with different dynamics and/or different parameters, it is possible that consensus is not reached, but a steady-state error may prevail - see e.g., Qin et al. (2018); Steur et al. (2016); Wang et al. (2015); Zhang et al. (2016). This may be referred to as practical consensus.

CONTACT: E. Panteley Email: panteley@lss.supelec.fr. This work was partially supported by the Dept. STITS of University of Paris Saclay and by the Government of Russian Federation (grant 08-08). 
The phenomenon is even more complex in the case of heterogeneous networks of systems that have no equilibria, such as oscillators. In this case it is more appropriate to speak of dynamic consensus which, roughly speaking, consists in all systems' trajectories synchronizing and reaching a common steady-state dynamical behaviour (as opposed to equilibria). Although the term was coined in (Panteley \& Loria, 2017), the concept is well documented in the literature of various research communities, including that of physics, computer science, biology, neurosciences etc. For instance, synchronization in neuronal activity has been recognized to cause neural disorders (Dunn \& Anderssen, 2011; Mormann et al., 2000), among which Parkinson's disease (Cagnan et al., 2009; Mallet et al., 2008). Understanding synchronization in disciplines such as biology, neurosciences, and medicine, has naturally led researchers to study of the collective behavior of networks of coupled nonlinear oscillators.

The first mathematical formulation of the synchronization problem for nonlinear oscillators is due to Andronov et al. (1987), which inspired the design of more general models of coupled oscillators, that include both phase and amplitude variations. Among these, the complex Stuart-Landau equation displays the amplitude equation derived from a general ordinary differential equation near an Andronov-Hopf bifurcation point (Sune et al., 2005). Networks of Stuart-Landau oscillators are used to model complex-systems behaviour in a wide range of applications; for instance, to describe chemical reaction diffusion systems (Ipsen et al., 1997), semiconductor lasers (Carr et al., 2006), electronic oscillators (Bergner et al., 2012), as well as neuro-physiological phenomena (Aoyagi, 1995).

One of the key questions in the study of networked oscillators is to determine how the synchronization and collective behavior is intertwined with the coupling strength. Assuming that in the limit (in terms of coupling gain) all oscillators have the same limit cycle, in the 1970s Y. Kuramoto proposed a reduced-order model which characterizes the limit-case behavior of such oscillators. The so-called Kuramoto model became one of the most popular models of phase oscillators in many disciplines including control theory. It exhibits cooperative phenomena such as frequency synchronization and phase-locking beyond a certain coupling strength — see e.g., Kuramoto (1975); Sakaguchi and Kuramoto (1986), and Strogatz (2000). The control problem was addressed, for instance, by Panteley, Loría, and El Ati (2015), as well as in (Selivanov et al., 2012) where communication delays are considered, and by Lehnert et al. (2014), under topological changes.

Now, there exist a range of tools in the literature to study stability properties of the limit cycle for a single Stuart-Landau oscillator (Andronov et al., 1987; Kuznetsov, 1998; Perko, 2000) as well as for networks of such oscillators (Karnatak et al., 2007; Teramae \& Tanaka, 2004) where Lyapunov-based techniques are used to study stability for a network of identical oscillators; see also Pham and Slotine (2007). If the coupling gain is finite and the network is heterogeneous the coupled Stuart-Landau oscillators are only frequency synchronized that is, the amplitudes of their oscillations do not coincide. Finding the synchronization frequency, however, is a challenging problem that remains open.

In this paper, which is the outgrowth of Panteley, Loría, and El Ati (2015), we present results on synchronization of Stuart-Landau oscillators with different frequencies of oscillation, under diffusive coupling. The analysis that we carry out is based on the framework laid in Panteley and Loria (2017), according to which synchronized behavior of heterogeneous networks consists in two parts: the so-called emergent dynamics and the synchronization of all oscillators relative to the emergent behavior. To 
that end, as in Olfati-Saber and Murray (2004) and many succeeding works, we employ a network model that is derived using a coordinate transformation. However, in place of using the Jordan decomposition of the Laplacian we base our transformation upon a matrix involving both the Laplacian and linear terms from the systems' dynamics. This is not innoccuous; such decomposition leads to the estimation of the synchronized oscillations frequency in function of the interconnection strength. There are, in addition, several technical specificities proper to Stuart-Landau oscillators, which are not covered in Panteley and Loria (2017). For instance, one technical difficulty in the study of Stuart-Landau oscillators is that the most classical Lyapunov stability tools are inapplicable since the equilibrium of the system form a compact disconnected set.

The rest of the paper is organized as follows. In the next section we describe the model of a single Stuart-Landau oscillator; in Section 3 we recall some important concepts related to the collective behavior of heterogenous networked systems. In Section 4 we describe the network structure under diffusive coupling and exhibit some fundamental properties of the interconnections' graph. In Section 5 we present a suitable model of the network dynamics in coordinates that exhibit the dichotomous character of the behavior of interconnected systems. In Section 6 we present our main results on stability of the collective behavior and, hence, in the synchronization for StuartLandau oscillators. The paper is wrapped up with some remarks in Section 8.

Notation. We denote a complex number $z \in \mathbb{C}$ as $z=z_{R}+\mathrm{i} z_{I}$ where $\mathrm{i}:=\sqrt{-1}$ and $z_{\mathrm{R}}, z_{\mathrm{I}} \in \mathbb{R}$ denote, respectively, the real and imaginary parts of $z$. We denote by $\bar{z}$ the complex conjugate of $z$, i.e., $\bar{z}=z_{\mathrm{R}}-\mathrm{i} z_{\mathrm{I}}$. Correspondingly, for complex vectors $\boldsymbol{z} \in \mathbb{C}^{N}, \boldsymbol{z}=\left[z_{1} \cdots z_{N}\right]^{\top}$ (where ${ }^{\top}$ denotes the usual transpose operator) and complex matrices $M \in \mathbb{C}^{N \times P}, M=\left[m_{i j}\right]$, we denote by $\overline{\boldsymbol{z}}$ and $\bar{M}$, their respective complex conjugates, i.e., $\overline{\boldsymbol{z}}=\left[\bar{z}_{1} \cdots \bar{z}_{N}\right]^{\top}$ and $\bar{M}=\left[\bar{m}_{i j}\right]$. We denote by ${ }^{*}$ the transpose conjugate operator for complex matrices and vectors hence, $\boldsymbol{z}^{*}=\left[\bar{z}_{1} \cdots \bar{z}_{N}\right]$. Also, we use $|\cdot|$ to denote $|z|=\bar{z} z$ and $|\boldsymbol{z}|=\boldsymbol{z}^{*} \boldsymbol{z}$. For a closed set $\mathcal{A} \subset \mathbb{C}^{n}$ and $\boldsymbol{x} \in \mathbb{C}^{n}$, we define $|\boldsymbol{x}|_{\mathcal{A}}:=\inf _{\boldsymbol{y} \in \mathcal{A}}|\boldsymbol{x}-\boldsymbol{y}|$.

\section{The generalized Stuart-Landau oscillator}

\subsection{The oscillator's model}

The Stuart-Landau dynamic equation is given by

$$
\dot{z}=-\nu|z|^{2} z+\mu z
$$

where $z \in \mathbb{C}$ denotes the state of the oscillator, $\nu, \mu \in \mathbb{C}$ are complex constant parameters defined as $\nu=\nu_{\mathrm{R}}+\mathrm{i} \nu_{\mathrm{I}}$ and $\mu=\mu_{\mathrm{R}}+\mathrm{i} \mu_{\mathrm{I}}$. The real part of $\mu$, denoted $\mu_{\mathrm{R}}$, determines the distance from the Andronov-Hopf bifurcation.

The subject of study in this paper is the stability of networks of oscillators (1). It is worth stressing that statements originally formulated for systems on Euclidean spaces may be used for systems whose solutions lay in the complex (hyper)plane. Indeed, in general, for a dynamical system $\dot{\boldsymbol{x}}=f(\boldsymbol{x})$ with complex state variables, $\boldsymbol{x} \in \mathbb{C}^{N}$, one can define stability in the sense of Lyapunov by decomposing $\boldsymbol{x}$ in its real and imaginary parts: $\boldsymbol{x}=\boldsymbol{x}_{\mathrm{R}}+\mathrm{i} \boldsymbol{x}_{\mathrm{I}} \in \mathbb{C}^{N}$. Then, we define the vector $\tilde{\boldsymbol{x}} \in \mathbb{R}^{2 N}$ as $\tilde{\boldsymbol{x}}:=\left[\begin{array}{ll}\boldsymbol{x}_{\mathrm{R}}^{\top} & \boldsymbol{x}_{\mathrm{I}}^{\top}\end{array}\right]^{\top}$. Note that, in particular, $|\tilde{\boldsymbol{x}}|^{2}=|\boldsymbol{x}|^{2}$. Then, provided that $f$ admits the decomposition $f(\boldsymbol{x}):=f_{\mathrm{R}}\left(\boldsymbol{x}_{\mathrm{R}}, \boldsymbol{x}_{\mathrm{I}}\right)+\mathrm{i} f_{\mathrm{I}}\left(\boldsymbol{x}_{\mathrm{R}}, \boldsymbol{x}_{\mathrm{I}}\right)$, we may re-express the dynamics 


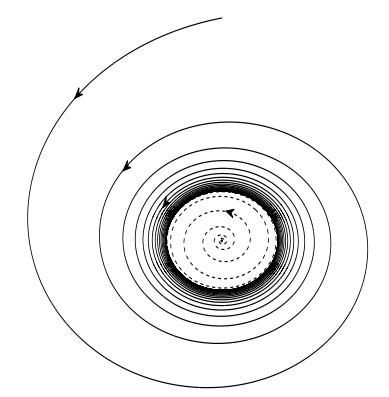

Figure 1. Trajectories of the Stuart-Landau oscillator on the complex plane in the case that $\nu_{\mathrm{R}}, \mu_{\mathrm{R}}>0$. The origin is unstable but all trajectories tend to a stable limit cycle with radius $r=\sqrt{\frac{\mu_{\mathrm{R}}}{\nu_{\mathrm{R}}}}$

of $\dot{\boldsymbol{x}}=f(\boldsymbol{x})$ in a $2 N$-dimensional Euclidean space, via

$$
\begin{aligned}
\dot{\boldsymbol{x}}_{\mathrm{R}} & =f_{\mathrm{R}}\left(\boldsymbol{x}_{\mathrm{R}}, \boldsymbol{x}_{\mathrm{I}}\right) \\
\dot{\boldsymbol{x}}_{\mathrm{I}} & =f_{\mathrm{I}}\left(\boldsymbol{x}_{\mathrm{R}}, \boldsymbol{x}_{\mathrm{I}}\right)
\end{aligned}
$$

and stability of the origin $\{\boldsymbol{x}=0\} \subset \mathbb{C}^{N}$ is equivalent to the stability of $\{\tilde{\boldsymbol{x}}=0\} \subset$ $\mathbb{R}^{2 N}$.

For Stuart-Landau oscillators (1) the stability analysis may be carried out using of polar coordinates, which are real. Let $z=r e^{i \varphi}$ then, the equations for the radial amplitude $r$ and the angular variable $\varphi$ can be decoupled into:

$$
\begin{aligned}
\dot{r} & =\mu_{\mathrm{R}} r-\nu_{\mathrm{R}} r^{3} \\
\dot{\varphi} & =\mu_{\mathrm{I}}-\nu_{\mathrm{I}} r^{2} .
\end{aligned}
$$

Note that the origin is unstable if $\mu_{\mathrm{R}}>0$. Also, if $\nu_{\mathrm{R}}<0$ the solutions may explode in finite time, while if $\nu_{\mathrm{R}}=0$ the oscillator becomes a simple first-order linear system. Thus, in the sequel, we assume that $\nu_{\mathrm{R}}>0$. Furthermore, when $\mu_{\mathrm{R}} \leq 0$, Equation (2a) has only one stable fixed point at $r=0$. Moreover, the latter is Lyapunov (globally exponentially) stable. In contrast to this, if $\mu_{\mathrm{R}}>0$, this equation has a stable fixed point $r=\sqrt{\frac{\mu_{\mathrm{R}}}{\nu_{\mathrm{R}}}}$, while $r=0$ becomes unstable. This implies that in this case the trajectories of the system converge to a circle of radius $r$, starting from initial conditions either inside or outside the circle. The curves

$$
\Gamma_{\alpha}=\sqrt{\frac{\mu_{\mathrm{R}}}{\nu_{\mathrm{R}}}}\left[\begin{array}{l}
\cos (t) \\
\sin (t)
\end{array}\right]
$$

define a limit cycle of the system - see the illustration in Figure 1. In this case, $z$ represents the position of the oscillator in the complex plane and $z(t)$ has a stable limit cycle of the amplitude $|z|=\sqrt{\frac{\mu_{\mathrm{R}}}{\nu_{\mathrm{R}}}}$ on which it moves at its natural frequency. The bifurcation of the limit cycle from the origin that appears at the value $\mu_{\mathrm{R}}=0$ is known in the literature as the Andronov-Hopf bifurcation. 


\subsection{Stability of the unforced Stuart-Landau oscillator}

As we have explained above, the set composed of a limit cycle plus the origin, that is,

$$
\mathcal{W}:=\left\{z \in \mathbb{C}:|z|=\sqrt{\frac{\mu_{\mathrm{R}}}{\nu_{\mathrm{R}}}}\right\} \bigcup\{z=0\}
$$

is invariant for the trajectories of the unforced oscillator (1). More precisely, the following theorem generalizes a statement from Pham and Slotine (2007) concerning the case of real coefficients, i.e., with $\nu_{\mathrm{R}}=1$ and $\nu_{\mathrm{I}}=0$.

Theorem 2.1. For the unforced Stuart-Landau oscillator, defined by Equation (1), the following statements hold true:

(1) if $\mu_{R}<0$ then the origin $z \equiv 0$ is globally exponentially stable;

(2) if $\mu_{R}>0$ then the limit cycle $\mathcal{W}_{1}=\left\{z \in \mathbb{C}:|z|=\sqrt{\mu_{R} / \nu_{R}}\right\}$ is almost globally asymptotically stable and the origin $\{z=0\}$ is anti-stable ${ }^{1}$. Moreover, in this case, the oscillation frequency on $\mathcal{W}_{1}$ is defined by

$$
\omega=\mu_{I}-\frac{\nu_{I}}{\nu_{R}} \mu_{R}
$$

Proof of Theorem 2.1

Proof of Item 1. Consider the Lyapunov function candidate $V(z)=|z|^{2}$. Using $|z|=\bar{z} z$ we see that the derivative of $V$ along trajectories of (1) yields

$$
\begin{aligned}
\dot{V}(z) & =\left[-\bar{\nu}|z|^{2} \bar{z}+\bar{\mu} \bar{z}\right] z+\bar{z}\left[-\nu|z|^{2} z+\mu z\right] \\
& =-(\nu+\bar{\nu})|z|^{4}+(\mu+\bar{\mu})|z|^{2} \\
& =-2 \nu_{\mathrm{R}}|z|^{4}+2 \mu_{\mathrm{R}}|z|^{2} .
\end{aligned}
$$

Now, since $\mu_{\mathrm{R}}<0$, we have $\dot{V}(z) \leq-\left|\mu_{\mathrm{R}}\right||z|^{2}$ for all $z \in \mathcal{C}$, so the origin is globally exponentially stable.

Proof of Item 2. Anti-stability of the origin, if $\mu_{\mathrm{R}}>0$, follows trivially by evaluating the total derivative of $V(z)=|z|^{2}$ along the trajectories of Equation (1) linearized around the origin, i.e., $\dot{z}=\mu z$. Indeed, locally, $\dot{V}=\mu_{\mathrm{R}} V$.

Next, to analyze the stability of the limit cycle $\mathcal{W}_{1}$, we introduce the Lyapunov function candidate

$$
V(z)=\frac{1}{4 \nu_{\mathrm{R}}}\left[|z|^{2}-\alpha\right]^{2}
$$

where $\alpha=\mu_{\mathrm{R}} / \nu_{\mathrm{R}}$. Notice that $V(z)=0$ for all $z \in \mathcal{W}_{1}$ and it is positive otherwise. Furthermore, evaluating the total derivative of $V$, along the solutions of (1), we obtain

$$
\begin{aligned}
\dot{V}(z) & =\frac{1}{2 \nu_{\mathrm{R}}}\left[|z|^{2}-\alpha\right][\dot{\bar{z}} z+\bar{z} \dot{z}] \\
& =-\left[|z|^{2}-\alpha\right]^{2}|z|^{2} .
\end{aligned}
$$

\footnotetext{
${ }^{1}$ That is, the poles of the linearized system have all positive real parts.
} 
We conclude that $\dot{V}$ is negative definite with respect to $\mathcal{W}_{1}$ that is, $\dot{V}<0$ for all $z \notin \mathcal{W}_{1}$ and $\dot{V}=0$ for all $z \in \mathcal{W}_{1}$. Since the origin is an anti-stable equilibrium point ${ }^{2}$, $\mathcal{W}_{1}$ is almost globally asymptotically stable.

It also follows that for any $r(0)>0, r(t) \rightarrow \sqrt{\mu_{\mathrm{R}} / \nu_{\mathrm{R}}}$ hence, after Equation $(2 \mathrm{~b})$ and the relation $\omega=\dot{\varphi}$, we have $\omega \rightarrow \mu_{\mathrm{I}}-\left(\nu_{\mathrm{I}} \mu_{\mathrm{R}}\right) / \nu_{\mathrm{R}}$.

\section{Synchronization of networked Stuart-Landau oscillators}

Let us consider now a network composed of $N$ heterogeneous Stuart-Landau oscillators that is, $N$ dynamical systems

$$
\begin{aligned}
\dot{z}_{i} & =f\left(z_{i}, \mu_{i}\right)+u_{i}, \quad i \in \mathcal{I}:=\{1, \ldots, N\} \\
f\left(z_{i}, \mu_{i}\right) & :=-\left|z_{i}\right|^{2} z_{i}+\mu_{i} z_{i}
\end{aligned}
$$

where $z_{i}, u_{i} \in \mathbb{C}$ are, respectively, the state and the input of $i$ th oscillator, $\mu_{i}=$ $\mu_{\mathrm{R} i}+\mathrm{i} \mu_{\mathrm{I} i} \in \mathbb{C}$ is a complex parameter that defines the asymptotic behavior of the $i$ th oscillator. Heterogeneity of the network is due to the parameters $\mu_{i} \in \mathbb{C}$ being different for each oscillator.

We assume that the oscillators are interconnected via diffusive coupling, which represents a static interaction between inputs and states of the oscillators, i.e., for the $i$ th oscillator the input is given by

$$
u_{i}=-\gamma\left[d_{i 1}\left(z_{i}-z_{1}\right)+d_{i 2}\left(z_{i}-z_{2}\right) \ldots+d_{i N}\left(z_{i}-z_{N}\right)\right], \quad d_{i j} \geq 0,
$$

where the scalar parameter $\gamma>0$ corresponds to the coupling strength.

In the particular case when oscillators are completely decoupled (i.e., $\gamma=0)$, all the oscillators in the network rotate at their individual (natural) frequencies with their own amplitudes. Actually, it was shown in Franci et al. (2012) that this individual behavior persists in the case of weak coupling (i.e., for small values of $\gamma$ ). The effect of network synchronization, which appears in the case of strong coupling may be of two types:

- Frequency synchronization: for sufficiently large values of $\gamma$ all the units tend asymptotically to oscillate at the same frequency, see e.g., Matthews et al. (1991).

- Phase locking: in addition to frequency synchronization the phase differences between the oscillators tend to be constant and are independent of the initial conditions.

In the case of a homogeneous and symmetric network, i.e., in which case $\mu_{i}=\mu_{j}$ for all $i, j \in \mathcal{I}$ and $L=L^{\top}$, all of the systems tend to oscillate at the same frequency and with zero phase differences if the coupling is strong. This effect, which is called complete network synchronization, is often described in terms of the asymptotically identical evolution of the units' motions. In other words, synchronization may be formulated as a problem of asymptotic stability of the synchronization manifold

$$
\mathcal{S}=\left\{z_{i} \in \mathbb{C}: z_{1}=z_{2}=\ldots=z_{N}\right\}
$$

\footnotetext{
${ }^{2}$ Solutions starting arbitrarily close to it, are repelled away.
} 
This problem may be broached using analysis tools developed for semi-passive, incrementally passive or incrementally input-output stable systems Franci et al. (2011); Jouffroy and Slotine (2004); Lohmiller and Slotine (2005); Pogromsky et al. (1999); Pogromsky and Nijmeijer (2001); Scardovi et al. (2009), among others.

The behavior of networks of systems with non-identical models, which is of concern here, is more complex due to the fact that the synchronization manifold $\mathcal{S}$ does not necessarily exist, but heterogeneous networks can exhibit some type of synchronization and collective behavior. In such case, it is natural to consider practical synchronization that is, to admit that, asymptotically, the differences between the units' motions are bounded and become smaller for larger values of the interconnection gain $\gamma$.

In Panteley and Loria (2017) was laid the basis of a framework for the study of practical synchronization of heterogeneous networks. In this paper, we pursue further the approach introduced in Panteley and Loria (2017) and extend it to obtain a better estimation of the synchronized system behavior. According to the latter, the behavior of interconnected heterogeneous systems coupled via diffusive coupling, may be studied via two separate properties: the stability of what we call the emergent dynamics and the synchronization errors of each of the units' motions relative to an averaged system, also called "mean-field" system. The emergent dynamics is an averaged model of the systems' dynamics regardless of the inputs while the mean-field oscillator's motion corresponds to the average of the units' motions and, as we shall see, its "steadystate" corresponds to the motion described by the emergent dynamics. For instance, in the classical paradigm of consensus of a collection of integrators,

$$
\dot{z}_{i}=u_{i}
$$

which is a particular case of our framework, the emergent dynamics is null while the mean field trajectory corresponds to the average $z_{m}(t)=(1 / N) \sum_{i=1}^{N} z_{i}(t)$. For a balanced graph, we know that all units reach consensus and the steady-state value is an equilibrium point corresponding to the average of the initial conditions -see Ren et al. (2007). In the framework introduced in Panteley and Loria (2017), the emergent dynamics possesses a stable attractor, in contrast to (the particular case of) an equilibrium point as is the case of (10). For Stuart-Landau oscillators, as we show, such attractor is a limit cycle.

Thus, the systems' behaviors over the network may be completely characterized via the stability of (the attractor of) the emergent dynamics and the asymptotic convergence of each unit's motion to the mean field's. More precisely, we shall show that in the case of general heterogeneous networks, with arbitrarily large values of the coupling gain $\gamma$, the analysis of the network behavior may be decomposed in two parts: the first relates to the dynamic behavior of the mean-field solutions $z_{m}(t)$. This is implemented by studying input to state stability of the average system dynamics with respect to a decomposable compact invariant set. The second part relates to the synchronization errors, the differences between each unit's trajectories, $z_{i}(t)$, and $z_{m}(t)$. This is captured by the stability of the synchronization errors manifold

$$
\mathcal{S}=\left\{\boldsymbol{e} \in \mathbb{C}^{N}: e_{1}=e_{2}=\ldots=e_{N}=0\right\}
$$

where $\boldsymbol{e}_{i}=z_{i}-z_{m}$.

To analyze stability properties of the set $\mathcal{S}$ we introduce the following definition of practical stability of a set, which is similar to that of practical stability of an equi- 
librium point -see Teel et al. (1999). Consider a parameterized system of differential equations

$$
\dot{x}=f(x, \varepsilon),
$$

where $x \in R^{n}$ is the state, $\varepsilon \in\left[\varepsilon^{*}, \infty\right)$ with $\varepsilon^{*}>0$ is a scalar parameter, and the function $f: \mathbb{R}^{n} \times\left[\varepsilon^{*}, \infty\right) \rightarrow \mathbb{R}^{n}$ is locally Lipschitz uniformly in $\varepsilon$. For such systems we recall the property of global practical uniform asymptotic stability with respect to closed, not necessarily compact, sets — cf. Panteley and Loria (2017).

Definition 3.1. For the system (12), we say that the closed set $\mathcal{A} \subset \mathbb{R}^{n}$ is practically uniformly asymptotically stable if there exists a closed set $\mathcal{D}$ such that $\mathcal{A} \subset \mathcal{D} \subset \mathbb{R}^{n}$ and:

(1) the system is forward complete for all $x_{\circ} \in \mathcal{D}$;

(2) for any given $\delta>0$ and $R>0$, there exist $\varepsilon^{*} \in\left[\varepsilon^{*}, \infty\right)$ such that, for all $\varepsilon \in\left[\varepsilon^{*}, \infty\right)$, there exists a class $\mathcal{K} \mathcal{L}$ function $\beta_{\varepsilon}$ such that, for all $\boldsymbol{x}_{\circ} \in \mathcal{D}$ satisfying $\left|\boldsymbol{x}_{\circ}\right|_{\mathcal{A}} \leq R$, we have

$$
\left|\boldsymbol{x}\left(t, \boldsymbol{x}_{\circ}, \varepsilon\right)\right|_{\mathcal{A}} \leq \delta+\beta_{\varepsilon}\left(\left|\boldsymbol{x}_{\circ}\right|_{\mathcal{A}}, t\right) .
$$

If $\mathcal{D}=\mathbb{R}^{n}$ then the set $\mathcal{A}$ is uniformly globally practically asymptotically stable.

\section{Network model transformation}

We assume that the network's graph is connected and undirected, in which case the interconnections between the nodes are defined by the adjacency matrix $\mathcal{D}:=\left[d_{i j}\right]_{i, j \in \mathcal{I}_{N}}$ where $d_{i j}=d_{j i}$ for all $i, j \in \mathcal{I}_{N}$. For simplicity we assume that the interconnections weights are real, i.e., $d_{i j} \in R$ for all $i, j \in \mathcal{I}_{N}$. Then, the corresponding Laplacian matrix is defined as

$$
L=\left[\begin{array}{cccc}
\sum_{i=2}^{N} d_{1 i} & -d_{12} & \ldots & -d_{1 N} \\
-d_{21} & \sum_{i=1, i \neq 2}^{N} d_{2 i} & \ldots & -d_{2 N} \\
\vdots & \vdots & \ddots & \vdots \\
-d_{N 1} & -d_{N 2} & \ldots & \sum_{i=1}^{N-1} d_{N i},
\end{array}\right]
$$

where all row sums are equal to zero. Since the the network is connected and undirected $L$ has exactly one eigenvalue (say, $\lambda_{1}$ ) equal to zero, while others are positive, i.e., $0=\lambda_{1}<\lambda_{2} \leq \ldots \leq \lambda_{N}$. Therefore, denoting by $\boldsymbol{z} \in \mathbb{C}^{N}$ the overall network's state, that is $\boldsymbol{z}=\left[z_{1}, \ldots, z_{N}\right]^{\top}$, using (7) and the expression for the diffusive coupling, (8), we see that the overall network dynamics can be described by the $N$ differential equations

$$
\dot{\boldsymbol{z}}=F(\boldsymbol{z})-\gamma L \boldsymbol{z}
$$

where the function $F: \mathbb{C}^{N} \rightarrow \mathbb{C}^{N}$ is given by

$$
F(\boldsymbol{z})=\left[f\left(z_{i}, \mu_{i}\right)\right]_{i \in \mathcal{I}} .
$$


In order to analyze the behavior of the solutions of (15) and according with the framework of Panteley and Loria (2017) we proceed to rewrite the system dynamics in new coordinates which exhibit the network emergent dynamics. We show that the synchronization properties may be deduced via an eigenvalue analysis of the linear part on the right-hand side of (15). To that end, we proceed to underline several structural properties of the networked system (15).

To start with, let

$$
C(\boldsymbol{z}):=\left[\begin{array}{cccc}
\left|\boldsymbol{z}_{1}\right|^{2} & 0 & \ldots & 0 \\
0 & \left|\boldsymbol{z}_{2}\right|^{2} & \ldots & 0 \\
\vdots & \ddots & \ddots & \vdots \\
0 & 0 & \ldots & \left|\boldsymbol{z}_{N}\right|^{2}
\end{array}\right] \text { and } \mathcal{M}:=\left[\begin{array}{cccc}
\mu_{1} & 0 & \ldots & 0 \\
0 & \mu_{2} & \ldots & 0 \\
\vdots & \ddots & \ddots & \vdots \\
0 & \ldots & 0 & \mu_{N}
\end{array}\right]
$$

then, we may rewrite the system (15) as

$$
\begin{aligned}
\dot{z} & =A_{\gamma} \boldsymbol{z}-C(\boldsymbol{z}) \boldsymbol{z} \\
A_{\gamma} & :=\mathcal{M}-\gamma L .
\end{aligned}
$$

The interest of representing the network dynamics as in (17) is that it enables us to study the behavior of the networked oscillators, following relatively simple arguments which rely on matrix and graph theories. Note that the eigenvalues of $A_{\gamma}$ approach those of $L$ (in absolute value) for large values of the interconnection gain $\gamma$. To see this, we express the matrix $A_{\gamma}$ as a "perturbed version" of the Laplacian, i.e.,

$$
A_{\gamma}=\gamma(-L+\varepsilon \mathcal{M}), \quad \varepsilon:=\frac{1}{\gamma}
$$

in which the parameter $\varepsilon=1 / \gamma$ may be rendered arbitrarily small by design. That is, for sufficiently large values of $\gamma$, we may use results on perturbation theory for matrices (see, e.g., Horn and Johnson (1985); Moro et al. (1997)) to characterize the eigenvalues and eigenvectors of $A_{\gamma}$ in terms of $\varepsilon$ and the eigenvalues and eigenvectors of the Laplacian L. In particular, (Moro et al., 1997, Theorem 2.1) as well as Horn and Johnson (1985), Wilkinson (1965) allow to estimate the eigenvalues of $A_{\gamma}$ in terms of those of $L, \mathcal{M}$ and $\varepsilon$. In general, a small perturbation of a generic matrix $A$ is denoted by

$$
A_{\varepsilon}=A_{0}+\varepsilon A_{1}, \quad \varepsilon \rightarrow 0
$$

so, if we denote by $\lambda_{1}\left(A_{0}\right)$ a simple eigenvalue of $A_{0}$ and by $\lambda_{1 \varepsilon}$ its induced perturbation, then, for sufficiently small $\varepsilon$, we may use the convergent power series representation

$$
\lambda_{\varepsilon}=\lambda_{1}+c_{1} \varepsilon+o(\varepsilon),
$$

where the coefficient of the first-order term, $c_{1} \varepsilon$, may be characterized as

$$
c_{1}=\frac{w^{\top} A_{1} v}{w^{\top} v}
$$


where $w$ and $v$ are normalized left and right eigenvectors of the unperturbed matrix $A_{0}$ associated to $\lambda_{1}$ hence, $|w|=|v|=1$. The statement also applies if the multiplicity of $\lambda_{1}$ is larger than one, provided that there exists a complete set of eigenvectors for the associated eigenspace Moro et al. (1997), Wilkinson (1965).

Now, for the system (17a) the Laplacian matrix $L$ is symmetric and corresponds to a connected graph hence, it is diagonalizable and there exists a real orthogonal matrix $U$ such that

$$
L=U\left[\begin{array}{lll}
\lambda_{1}(L) & & \\
& \ddots & \\
& & \lambda_{N}(L)
\end{array}\right] U^{\top}
$$

where, we recall that $\lambda_{1}(L)=0$. Moreover, since $L$ is symmetric its left and right eigenvectors corresponding to $\lambda_{1}(L)=0$ coincide and are given by

$$
w=v=\frac{1}{N} \mathbf{1}, \quad \mathbf{1}:=\left[\begin{array}{lll}
1 & \cdots & 1
\end{array}\right]^{\top} .
$$

Thus, by assimilating $A_{\varepsilon}$ in $(18)$ to $(-L+\varepsilon \mathcal{M})$ hence, $A_{0}$ to $-L$ and $A_{1}$ to $\mathcal{M}$, we see from (20), that

$$
c_{1}=\frac{1}{N} \mathbf{1}^{\top} \mathcal{M} \mathbf{1}=\frac{1}{N} \sum_{i=1}^{N} \mu_{i}
$$

and we deduce that the eigenvalues of $A_{\gamma}$ may be approximated, via (19), as

$$
\begin{aligned}
\lambda_{1}\left(A_{\gamma}\right) & =\gamma\left[-\lambda_{1}(L)+c_{1} \varepsilon+o(\varepsilon)\right] \\
& =\gamma\left[c_{1} \frac{1}{\gamma}+o\left(\frac{1}{\gamma}\right)\right] \\
& =\frac{1}{N} \sum_{i=1}^{N} \mu_{i}+O\left(\frac{1}{\gamma}\right)
\end{aligned}
$$

We conclude that $\lambda_{1}\left(A_{\gamma}\right)$ is bounded as a function of $\gamma$ and it converges to $\frac{1}{N} \sum_{i=1}^{N} \mu_{i}$ as the coupling strength $\gamma \rightarrow \infty$. Moreover, for all $j \in\{2, \ldots, N\}$ we have

$$
\lambda_{j}\left(A_{\gamma}\right)=-\gamma \lambda_{j}(L)+c_{1}+O(\varepsilon)
$$

where $c_{1}$ was defined in (20), hence, the eigenvalues of $A_{j}$ are proportional to $\gamma$ and, since $\Re e\left[\lambda_{j}(L)\right]>0$, we have $\Re e\left[\lambda_{j}\left(A_{\gamma}\right)\right] \rightarrow-\infty$ as $\gamma \rightarrow \infty$.

On the other hand, we underline that $A_{\gamma} \in \mathbb{C}^{N \times N}$ is complex symmetric, i.e., $A_{\gamma}=A_{\gamma}^{\top}$ and for any symmetric complex matrix $M$ there exists a complex orthogonal 
matrix $T$, i.e., satisfying ${ }^{3} T^{-1}=T^{\top}$, such that $T^{\top} M T$ has the block-diagonal form

$$
\left[\begin{array}{cccc}
M_{1} & 0 & 0 & \ldots \\
0 & M_{2} & 0 & \ldots \\
0 & 0 & M_{3} & \ldots \\
\cdots & \ldots & \ldots & \ldots
\end{array}\right]
$$

where each block $M_{k}$ is either scalar, if the eigenvalue is simple, or $M_{k}=\lambda_{k} I+\tilde{M}$ where $\tilde{M} \in \mathbb{C}^{q \times q}$, if the eigenvalue has multiplicity $q$, and the eigenvalues of $\tilde{M}_{k}$ equal to zero (see e.g., Craven (1969); Horn and Johnson (1985)).

In view of the above, the following assumption is little restrictive. The first part follows by construction as well as from the properties of the Laplacian for undirected graphs, which satisfies (21). The second part, that the largest eigenvalue of $A_{\gamma}$ is simple, follows after the observation that for large values of the interconnection gains, the eigenvalues of $A_{\gamma}$ approach those of $L$.

Assumption 1. There exists a number $\gamma^{*}>0$ and, for each $\gamma \geq \gamma^{*}$, a diagonal matrix $\Lambda_{\gamma} \in \mathbb{C}^{N \times N}$, whose elements corresponds to the eigenvalues of $A_{\gamma}$, and a complex orthogonal matrix $V_{\gamma} \in \mathbb{C}^{N \times N}$, i.e., such that

$$
V_{\gamma}^{\top} V_{\gamma}=I_{N}
$$

and the matrix $A_{\gamma}$ defined in (17b) may be factorized as

$$
A_{\gamma}=V_{\gamma} \Lambda_{\gamma} V_{\gamma}^{-1}
$$

Moreover, there exists $k \leq N$ such that $\Re e\left[\lambda_{k}\right]>\max _{j \in \mathcal{I} \cap j \neq k} \Re e\left[\lambda_{j}\right]$.

Without loss of generality, in what follows we assume that the eigenvalues of $A_{\gamma}$ are ordered in decreasing order, that is, $\lambda_{1}\left(A_{\gamma}\right)$ has the largest real part and $\Re e\left[\lambda_{1}\right]>$ $\Re e\left[\lambda_{2}\right] \geq \ldots \geq \Re e\left[\lambda_{N}\right]$.

We remark that the $i$ th column of the matrix $V_{\gamma}$ corresponds to the right eigenvector, denoted $\vartheta_{\mathbf{r}_{i}}$, associated to the $i$ th eigenvalue of $A_{\gamma}$. Correspondingly, we denote by $\vartheta_{\ell_{i}}$ the $i$ th left eigen-vector, which corresponds to the $i$ th row of $V_{\gamma}^{\top}$. Therefore, we have

$$
\begin{aligned}
& A_{\gamma} \vartheta_{\mathbf{r}_{i}}=\lambda_{i}\left(A_{\gamma}\right) \vartheta_{\mathbf{r}_{i}} \\
& \vartheta_{\ell_{i}} A_{\gamma}=\lambda_{i}\left(A_{\gamma}\right) \vartheta_{\ell_{i}}
\end{aligned}
$$

Moreover, due to the complex-orthogonality of $V_{\gamma}$, expressed by Equation (24), we have

$$
\left[\vartheta_{\mathbf{r}}\right]^{2}:=\vartheta_{\mathbf{r}}^{\top} \vartheta_{\mathbf{r}}=1, \quad \vartheta_{\ell_{i}}=\bar{\vartheta}_{\mathbf{r}_{i}}
$$

Another crucial feature of (17) is that it leads to a new representation of the dynamics, which is reminiscent of that of a homogeneous network. A fundamental fact that we shall exhibit is that $A_{\gamma}$ possesses properties similar to those of the Laplacian $L$. To

\footnotetext{
${ }^{3}$ We stress the difference between complex orthogonal matrices, which satisfy $T^{-1}=T^{\top}$, and unitary matrices satisfying $T^{-1}=T^{*}$
} 
see this, we proceed to decompose the matrix $A_{\gamma}$ as follows. According to Assumption 1 the matrix matrix $\Lambda_{\gamma}$ is diagonal hence, we may introduce $\Lambda_{1}, \Lambda_{2}$ such that

$$
\begin{aligned}
& \Lambda=\Lambda_{1}+\Lambda_{2}, \quad \Lambda_{1}:=\lambda_{1}\left(A_{\gamma}\right) I \\
& \Lambda_{2}:=\left(\begin{array}{cccc}
0 & 0 & \cdots & 0 \\
\vdots & \lambda_{2}\left(A_{\gamma}\right)-\lambda_{1}\left(A_{\gamma}\right) & 0 & \vdots \\
0 & 0 & \ddots & 0 \\
0 & \cdots & 0 & \lambda_{N}\left(A_{\gamma}\right)-\lambda_{1}\left(A_{\gamma}\right)
\end{array}\right)
\end{aligned}
$$

Notice that since $\gamma>\gamma^{*},(N-1)$ non-zero eigenvalues of the matrix $\Lambda_{2}$ have negative real parts and, moreover, for all $i \in\{2, \ldots, N\}$ we have $\Re e\left[\lambda_{i}\left(\Lambda_{2}\right)\right] \rightarrow-\infty$ as $\gamma \rightarrow+\infty$ - see (22) and (23).

Using these notations we can rewrite the matrix $A_{\gamma}$ as

$$
A_{\gamma}=V_{\gamma} \Lambda_{1} V_{\gamma}^{\top}+V_{\gamma} \Lambda_{2} V_{\gamma}^{\top}=\lambda_{1}\left(A_{\gamma}\right) I+D
$$

where $D=V_{\gamma} \Lambda_{2} V_{\gamma}^{\top}$. The interest of the matrix $D$ is that it depends on the systems' parameters $\mu_{i}$ but it inherits the properties of the Laplacian matrix; indeed, in view of the definition of $\Lambda_{2}$ and Assumption 1 we have $D \leq 0$ and, moreover, it has one zero eigenvalue and $N-1$ eigenvalues of this matrix have negative real parts. As a matter of fact, for all $i \in\{2, \ldots, N\}$, we have

$$
\lambda_{i}(D)=\lambda_{i}\left(\Lambda_{2}\right), \Re e\left[\lambda_{i}\left(\Lambda_{2}\right)\right] \rightarrow-\infty \quad \text { as } \quad \gamma \rightarrow+\infty .
$$

Moreover, the right eigenvectors $\vartheta_{\mathbf{r}_{i}}$ associated to the eigenvalues $\lambda_{i}\left(A_{\gamma}\right)$ of $A_{\gamma}$ are also the respective right eigenvectors associated to the eigenvalues of $D$ and $\lambda_{i}(D)=$ $\lambda_{i}\left(A_{\gamma}\right)-\lambda_{1}\left(A_{\gamma}\right)$. Indeed, we have, for each $i \in \mathcal{I}, D \vartheta_{\mathbf{r}_{i}}=V_{\gamma} \Lambda_{2} V_{\gamma}^{\top} \vartheta_{\mathbf{r}_{i}}$. On the other hand, since $\vartheta_{\mathbf{r}_{i}}^{\top} \vartheta_{\mathbf{r}_{j}}=0$ for all $i \neq j$ and $\vartheta_{\mathbf{r}_{i}}^{\top} \vartheta_{\mathbf{r}_{i}}=1$, we have $D \vartheta_{\mathbf{r}_{i}}=\vartheta_{\mathbf{r}_{i}} \lambda_{i}(D)$. Clearly, since $\lambda_{1}(D)=0$ we also have $D \vartheta_{\mathbf{r}_{1}}=0$.

The overall conclusion is that the networked system (17a) may be expressed in the alternative form

$$
\dot{z}=\left[\lambda_{1} I-C(\boldsymbol{z})\right] \boldsymbol{z}+D \boldsymbol{z}
$$

which is no more than an alternative manner of writing the equations of motion of the interconnected heterogeneous oscillators, (15). The interest of this representation is that it is reminiscent of a network in which the oscillators have equal parameters $\mu_{i}$. Indeed, notice that the dynamics equation for a network (7), (8) with $\mu_{i}=\mu_{j}=\mu$ for all $i, j \in \mathcal{I}$ takes the form

$$
\dot{z}=[\mu I-C(\boldsymbol{z})] \boldsymbol{z}-\gamma L \boldsymbol{z}
$$

Thus, the fact that $D$ inherits the properties of the Laplacian matrix enables us, to some extent, to interpret the original network of heterogeneous oscillators as a network where all the nodes have identical dynamics. 


\section{Network dynamics}

\subsection{Coordinate transformation}

Even though the diagonalizability of $A_{\gamma}$ allows us to reinterpret the network's equation of motion as that of a homogeneous network, the significance of this property is well beyond pure analytic interest. As we show next, it also allows to exhibit the emergent dynamics, which is at the core of the networked systems behavior and, therefore, at the basis the analysis framework Panteley and Loria (2017).

To see this clearer, we proceed to represent the system (17) in a coordinates frame whose first coordinate corresponds to a certain "average" of all the units' states. The rest of the coordinates, which stem naturally from this representation, correspond to the synchronization errors. Such coordinate transformation, which is defined upon the transformation matrix $V_{\gamma}$ simplifies considerably the analysis of the networked system. Let

$$
\tilde{\boldsymbol{z}}=V_{\gamma}^{\top} \boldsymbol{z}
$$

and let $\widetilde{V}_{\gamma}:=\left[\vartheta_{\mathbf{r}_{2}} \cdots \vartheta_{\mathbf{r}_{\mathrm{N}}}\right]$ then,

$$
\tilde{\boldsymbol{z}}=\left[\begin{array}{c}
\tilde{\boldsymbol{z}}_{1} \\
\tilde{\boldsymbol{z}}_{2}
\end{array}\right]=\left[\begin{array}{c}
\vartheta_{\mathbf{r}_{1}}^{\top} \\
\widetilde{V}_{\gamma}^{\top}
\end{array}\right] \boldsymbol{z} .
$$

From Section 4 we know that $\lambda_{1}\left(A_{\gamma}\right) \rightarrow \lambda_{1}(L)$ as $\gamma \rightarrow \infty$ and, $\vartheta_{\mathbf{r}_{1}}$, which corresponds to the first right eigenvector of both, $A_{\gamma}$ and $D$, satisfies $\vartheta_{\mathbf{r}_{1}} \rightarrow \mathbf{1}$, as $\gamma \rightarrow \infty$. It follows that in the limit the coordinate $\tilde{\boldsymbol{z}}_{1}$ converges to the vector

$$
z_{e}=\frac{1}{N} \sum_{i=1}^{N} z_{i}
$$

which in the literature on nonlinear oscillators is referred to as the state of the averaged or mean-field oscillator -see Belhaq and Houssni (2000); Rosenblum and Pikovsky (2004). In other words, $\tilde{\boldsymbol{z}}_{1}$ may be regarded as a weighted average of the units' states $z_{i}$.

Next, let us consider the rest of the coordinates in $\tilde{\boldsymbol{z}}$, i.e., the vector $\tilde{\boldsymbol{z}}_{2}=\widetilde{V}_{\gamma}^{\top} \boldsymbol{z}$. From (24) we have $V_{\gamma}^{\top}=V_{\gamma}^{-1}$, so

$$
\widetilde{V}_{\gamma} \widetilde{V}_{\gamma}^{\top}=I_{N}-\vartheta_{\mathbf{r}_{1}} \vartheta_{\mathbf{r}_{1}}^{\top}
$$

and, pre-multiplying $\tilde{\boldsymbol{z}}_{2}$ by $\widetilde{V}_{\gamma}$ and using (31) we see that $\overline{\boldsymbol{z}}_{2}$ equals to zero if and only if $\boldsymbol{z}=\vartheta_{\mathbf{r}_{1}} \tilde{\boldsymbol{z}}_{1}$ or, equivalently, if the synchronization error $\boldsymbol{e} \in \mathbb{C}^{N}$ defined as $\boldsymbol{e}=\boldsymbol{z}-\vartheta_{\mathbf{r}_{1}} \tilde{\boldsymbol{z}}_{1}$ equals to zero. That is, $\tilde{\boldsymbol{z}}_{2}$ constitutes a natural measure of synchrony among the oscillators in the network; it corresponds to the synchrony between each oscillator and the network mean-field.

Thus, the behavior of the networked systems interconnected via diffusive coupling 
is naturally and completely captured by the states

$$
\begin{aligned}
z_{m} & :=\vartheta_{\mathbf{r}_{1}}^{\top} \boldsymbol{z} \\
\boldsymbol{e} & :=\boldsymbol{z}-\vartheta_{\mathbf{r}_{1}} z_{m} .
\end{aligned}
$$

By using these coordinates we decompose the analysis of the network behavior in two distinct parts: the first, pertains to the "average" behavior of the network and the second, to the synchronization of the units. Notice that due to the definition of $\vartheta_{\mathbf{r}_{1}}$ here, in contrast to Panteley and Loria (2017), the definition of the average behavior state $z_{m}$ depends explicitly on the coupling strength $\gamma$. For heterogeneous networks we have that $z_{m} \rightarrow z_{e}$ asymptotically, as $\gamma \rightarrow \infty$, while for homogeneous networks $z_{m}=z_{e}$. Moreover, in view of the heterogeneity of the network, one can only expect that the synchronization errors become arbitrarily small for arbitrarily large values of the interconnection gain $\gamma$.

Remark 1. The vector $\boldsymbol{e}$ corresponds to the errors between each oscillator with state $z_{i}$ and the scaled and rotated mean-field oscillator, with state $z_{m}$. In general, the vector $\vartheta_{\mathbf{r}_{1}}$ does not necessarily have only rotational components since some of its coefficients may be different from one. However, in the limit, as $\gamma \rightarrow \infty$, we have $\vartheta_{\mathbf{r}_{1}} \rightarrow \mathbf{1}$ so for sufficiently large values of $\gamma$, the elements of $\vartheta_{\mathbf{r}_{1}}$ converge to $e^{\mathrm{i} \varphi_{j}}$ where $\varphi_{j} \in \mathbb{R}$. Thus, for sufficiently large values of $\gamma$, the right eigen-vector $\vartheta_{\mathbf{r}_{1}}$ may be approximated as a vector of rotations which correspond to the phase difference between the interconnected oscillators and the average oscillator.

In what follows, we derive the dynamics equations corresponding to $z_{m}$ and $\boldsymbol{e}$.

\subsection{Dynamics of the averaged oscillator}

We differentiate on both sides of (32a) and use the network dynamics equation (29) to obtain

$$
\begin{aligned}
\dot{z}_{m} & =\vartheta_{\mathbf{r}_{1}}^{\top}\left[\left(\lambda_{1}\left(A_{\gamma}\right) I-C(\boldsymbol{z})\right) \boldsymbol{z}+D \boldsymbol{z}\right] \\
& =\lambda_{1}\left(A_{\gamma}\right) z_{m}-\vartheta_{\mathbf{r}_{1}}^{\top} C(\boldsymbol{z}) \boldsymbol{z}+\vartheta_{\mathbf{r}_{1}}^{\top} D \boldsymbol{z}
\end{aligned}
$$

however, since $\vartheta_{\mathbf{r}_{1}}$ is an eigen-vector (also) associated to $\lambda_{1}(D)$, the last term on the right-hand side of (33) equals to zero. We proceed to rewrite the rest of the right-hand side of (33) in terms of $z_{m}$ and $\boldsymbol{e}$. From (32b), we have $C(\boldsymbol{z}) \boldsymbol{z}=C(\boldsymbol{z})\left[\boldsymbol{e}+\vartheta_{\mathbf{r}_{1}} z_{m}\right]$. Next let us introduce the operator $\Gamma$ defined as

$$
\Gamma(\boldsymbol{z}):=\left[\begin{array}{cccc}
z_{1} & 0 & \ldots & 0 \\
0 & z_{2} & \ldots & 0 \\
\vdots & \ddots & \ddots & \vdots \\
0 & \ldots & 0 & z_{N}
\end{array}\right]
$$


notice that $I_{N}=\Gamma(\mathbf{1}), \Gamma(\boldsymbol{x}) \boldsymbol{y}=\Gamma(\boldsymbol{y}) \boldsymbol{x}$ and $\Gamma(\boldsymbol{x})-\Gamma(\boldsymbol{y})=\Gamma(\boldsymbol{x}-\boldsymbol{y})$ for all $\boldsymbol{x}, \boldsymbol{y} \in \mathbb{C}^{N}$. Also, $C(\boldsymbol{z})=\Gamma(\boldsymbol{z})^{*} \Gamma(\boldsymbol{z})$ where $\Gamma^{*}$ denotes the conjugate transpose of $\Gamma$, hence,

$$
\begin{aligned}
& C(\boldsymbol{z}) \boldsymbol{z}=C(\boldsymbol{z}) \boldsymbol{e}+\Gamma(\boldsymbol{z})^{*} \Gamma(\boldsymbol{z}) \vartheta_{\mathbf{r}_{1}} z_{m} \pm \Gamma(\boldsymbol{z})^{*}\left[\Gamma\left(\vartheta_{\mathbf{r}_{1}} z_{m}\right) \vartheta_{\mathbf{r}_{1}} z_{m}\right] \\
& =C(\boldsymbol{z}) \boldsymbol{e}+\Gamma(\boldsymbol{z})^{*}\left[\Gamma\left(\boldsymbol{z}-\vartheta_{\mathbf{r}_{1}} z_{m}\right) \vartheta_{\mathbf{r}_{1}} z_{m}+\Gamma\left(\vartheta_{\mathbf{r}_{1}} z_{m}\right) \vartheta_{\mathbf{r}_{1}} z_{m}\right] \\
& =C(\boldsymbol{z}) \boldsymbol{e}+\Gamma(\boldsymbol{z})^{*} \Gamma\left(\vartheta_{\mathbf{r}_{1}} z_{m}\right) \boldsymbol{e}+\Gamma(\boldsymbol{z})^{*} \Gamma\left(\vartheta_{\mathbf{r}_{1}} z_{m}\right) \vartheta_{\mathbf{r}_{1}} z_{m} \pm \Gamma\left(\overline{\vartheta_{\mathbf{r}_{1}} z_{m}}\right) \Gamma\left(\vartheta_{\mathbf{r}_{1}} z_{m}\right) \vartheta_{\mathbf{r}_{1}} z_{m}
\end{aligned}
$$

where $\overline{\vartheta_{\mathbf{r}_{1}} z_{m}}=\left[\bar{\vartheta}_{\mathbf{r}_{11}} \bar{z}_{m} \cdots \bar{\vartheta}_{\mathbf{r}_{1 \mathrm{~N}}} \bar{z}_{m}\right]^{\top}$ and we used $\Gamma(\boldsymbol{z})^{*}=\Gamma(\overline{\boldsymbol{z}})$. Therefore, using $\overline{\boldsymbol{e}}=\overline{\boldsymbol{z}}-\overline{\vartheta_{\mathbf{r}_{1}} z_{m}}$, the linearity of $\Gamma$ and $\left|z_{m}\right|^{2}=\bar{z}_{m} z_{m}$, we obtain

$$
\begin{aligned}
C(\boldsymbol{z}) \boldsymbol{z} & =\left[C(\boldsymbol{z})+\Gamma(\boldsymbol{z})^{*} \Gamma\left(\vartheta_{\mathbf{r}_{1}} z_{m}\right)\right] \boldsymbol{e}+\Gamma(\overline{\boldsymbol{e}}) \Gamma\left(\vartheta_{\mathbf{r}_{1}} z_{m}\right) \vartheta_{\mathbf{r}_{1}} z_{m}+\Gamma\left(\bar{\vartheta}_{\mathbf{r}_{1}}\right) \Gamma\left(\vartheta_{\mathbf{r}_{1}}\right) \vartheta_{\mathbf{r}_{1}}\left|z_{m}\right|^{2} z_{m} \\
& =\left[C(\boldsymbol{z})+\Gamma(\boldsymbol{z})^{*} \Gamma\left(\vartheta_{\mathbf{r}_{1}} z_{m}\right)\right] \boldsymbol{e}+\Gamma\left(\left[\vartheta_{\mathbf{r}_{11}}^{2} \cdots \vartheta_{\mathbf{r}_{1 N}}^{2}\right]^{\top}\right)\left(z_{m}\right)^{2} \overline{\boldsymbol{e}}+\Gamma\left(\bar{\vartheta}_{\mathbf{r}_{1}}\right) \Gamma\left(\vartheta_{\mathbf{r}_{1}}\right) \vartheta_{\mathbf{r}_{1}}\left|z_{m}\right|^{2} z_{m} .
\end{aligned}
$$

Using the latter in (33), we obtain

$$
\dot{z}_{m}=\lambda_{1} z_{m}-\alpha\left|z_{m}\right|^{2} z_{m}-\vartheta_{\mathbf{r}_{1}}^{\top}\left[C(\boldsymbol{z})+\Gamma(\boldsymbol{z})^{*} \Gamma\left(\vartheta_{\mathbf{r}_{1}} z_{m}\right)\right] \boldsymbol{e}-\vartheta_{\mathbf{r}_{1}}^{\top} \Gamma\left(\left[\vartheta_{\mathbf{r}_{11}}^{2} \cdots \vartheta_{\mathbf{r}_{1 N}}^{2}\right]^{\top}\right)\left(z_{m}\right)^{2} \overline{\boldsymbol{e}}
$$

where

$$
\alpha=\vartheta_{\mathbf{r}_{1}}^{\top} \Gamma\left(\bar{\vartheta}_{\mathbf{r}_{1}}\right) \Gamma\left(\vartheta_{\mathbf{r}_{1}}\right) \vartheta_{\mathbf{r}_{1}}
$$

hence,

$$
\begin{aligned}
\dot{z}_{m} & =\left[\lambda_{1}-\alpha\left|z_{m}\right|^{2}\right] z_{m}+f_{m}\left(z_{m}, \boldsymbol{e}\right) \\
f_{m}\left(z_{m}, \boldsymbol{e}\right) & :=-\vartheta_{\mathbf{r}_{1}}^{\top}\left[C(\boldsymbol{z})+\Gamma(\boldsymbol{z})^{*} \Gamma\left(\vartheta_{\mathbf{r}_{1}} z_{m}\right)\right] \boldsymbol{e}-\vartheta_{\mathbf{r}_{1}}^{\top} \Gamma\left(\left[\vartheta_{\mathbf{r}_{11}}^{2} \cdots \vartheta_{\mathbf{r}_{1 \mathrm{~N}}}^{2}\right]^{\top}\right)\left(z_{m}\right)^{2} \overline{\boldsymbol{e}}
\end{aligned}
$$

Notice that $f_{m} \equiv 0$ if $|\boldsymbol{e}|^{2}=\overline{\boldsymbol{e}}^{\top} \boldsymbol{e}=0$ that is, if synchronization is achieved asymptotically the dynamics of the average unit, (35), converges to the emergent dynamics

$$
\dot{z}_{e}=\left[\lambda_{1}-\alpha\left|z_{e}\right|^{2}\right] z_{e}
$$

Hence, a reasonably good measure of stability of the solutions of (35a) is that with respect to invariant sets for the solutions of (36).

Remark 2. We stress that even though the interconnection gain $\gamma$ does not appear explicitly in the right-hand side of (35), we see from (22) that $\lambda_{1}\left(A_{\gamma}\right)=c+O\left(\frac{1}{\gamma}\right)$, where the constant $c$ depends only on the matrix $\mathcal{M}$. That is, in contrast to Panteley and Loria (2017), the emergent dynamics equation (36) depends on the coupling strength $\gamma$ hence, it better approximates the network's synchronized behavior.

\subsection{Dynamics of the synchronization errors}

Next, we derive the dynamics equation corresponding to the synchronization error (32b). To that end, let us start by introducing the matrix

$$
P:=\left(I-\vartheta_{\mathbf{r}_{1}} \vartheta_{\mathbf{r}_{1}}^{\top}\right)
$$


hence, we have $\boldsymbol{e}=P \boldsymbol{z}$. Next, differentiating on both sides of the latter and using (29) we obtain the error dynamics of $\boldsymbol{e}$,

$$
\dot{\boldsymbol{e}}=P D \boldsymbol{z}+P\left[\lambda_{1}\left(A_{\gamma}\right) I-C(\boldsymbol{z})\right] \boldsymbol{z} .
$$

Now, since $\vartheta_{\mathbf{r}_{1}}$ is a right eigen-vector associated to $\lambda_{1}(D)=0$, it follows that $D P=$ $D=P D$. Indeed, on one hand, $D$ and $P$ are both symmetric and, on the other, $D P=D-D \vartheta_{\mathbf{r}_{1}} \vartheta_{\mathbf{r}_{1}}^{\top}$ and $D \vartheta_{\mathbf{r}_{1}}=0$. Therefore, $P D \boldsymbol{z}=P D P \boldsymbol{z}$ and, since $\boldsymbol{e}=P \boldsymbol{z}$, we obtain $P D \boldsymbol{z}=P D \boldsymbol{e}$. It follows from this and (37) that

$$
\begin{aligned}
\dot{\boldsymbol{e}} & =\left[P D+\lambda_{1}\left(A_{\gamma}\right) I\right] \boldsymbol{e}-P C(\boldsymbol{z}) \boldsymbol{z} . \\
& =\left[D+\lambda_{1}\left(A_{\gamma}\right) I\right] \boldsymbol{e}-P C\left(\boldsymbol{e}+\vartheta_{\mathbf{r}_{1}} z_{m}\right)\left[\boldsymbol{e}+\vartheta_{\mathbf{r}_{1}} z_{m}\right]
\end{aligned}
$$

Thus, Equations (35) and (38) completely define the dynamics of the networked oscillators interconnected via diffusive coupling and in coordinates meaningful for our purposes of analysis. The next section is devoted to the stability analysis of the solutions of these equations, which we regroup for convenience:

$$
\begin{aligned}
\dot{z}_{m} & =\left[\lambda_{1}-\alpha\left|z_{m}\right|^{2}\right] z_{m}+f_{m}\left(z_{m}, \boldsymbol{e}\right) \\
\dot{\boldsymbol{e}} & =\left[D+\lambda_{1} I\right] \boldsymbol{e}-P C\left(\boldsymbol{e}+\vartheta_{\mathbf{r}_{1}} z_{m}\right)\left[\boldsymbol{e}+\vartheta_{\mathbf{r}_{1}} z_{m}\right] .
\end{aligned}
$$

We investigate two different properties. Firstly, we establish a bound on the synchronization errors $\boldsymbol{e}$. Then, the second part relates to the stability of the natural attractor of the emergent dynamics (36), which corresponds to the nominal part of (39a). Notice that this is tantamount to studying the robust stability of an isolated unforced StuartLandau equation; more precisely, input-to-state stability with respect to invariant sets of (36) and the input $\boldsymbol{e}$. In other words, in a first stage, we analyze the behavior of the solutions of $(39 \mathrm{~b})$ and in a second stage, those of (39a).

\section{Networked systems' stability}

\subsection{Ultimate boundedness of solutions}

As a preliminary but fundamental step in the analysis of Equations (39) we formulate conditions that ensure that the trajectories of the networked diffusively-coupled Stuart-Landau oscillators, as described by (15) and equivalently by (39), are globally ultimately bounded that is there exists a positive constant $T$ such that, for all $\boldsymbol{z}_{\circ} \in \mathbb{C}$

$$
\left|\boldsymbol{z}\left(t, \boldsymbol{z}_{\circ}\right)\right| \leq c \quad \forall t \geq T .
$$

This property may be established for any interconnection gain $\gamma>0$, using simple Lyapunov arguments - cf. Matthews et al. (1991); Pham and Slotine (2007). In a general context, conditions for ultimate boundedness are given, e.g., in (Khalil, 2002, Theorem 4.18).

Proposition 6.1. Consider the system (15), (16) and let the graph of the network be 
undirected and connected. Then, the solutions are globally ultimately bounded and

$$
\begin{aligned}
\left|\boldsymbol{z}\left(t, \boldsymbol{z}_{\circ}\right)\right| & \leq \sqrt{2 \bar{\mu} N}, \quad \forall t \geq T \\
\bar{\mu} & =\max _{i \in \mathcal{I}}\left\{\mu_{\mathrm{R} i}, 0\right\} .
\end{aligned}
$$

Proof. Consider the Lyapunov function candidate $V(\boldsymbol{z})=\boldsymbol{z}^{*} \boldsymbol{z}$; it is clear that $V$ is positive definite, decrescent and radially unbounded.

Now, evaluating the total derivative of $V$ along the system's trajectories, using the symmetry of the Laplacian $L$ and the fact that all the eigenvalues of the latter are non-negative, we obtain

$$
\dot{V}(\boldsymbol{z}) \leq \boldsymbol{z}^{*} F(\boldsymbol{z})+F(\boldsymbol{z})^{*} \boldsymbol{z}
$$

where

$$
F(\boldsymbol{z})=-C(\boldsymbol{z}) \boldsymbol{z}+\mathcal{M} \boldsymbol{z}, \quad F(\boldsymbol{z})^{*}=-\boldsymbol{z}^{*} C(\boldsymbol{z})+\boldsymbol{z}^{*} \mathcal{M}^{*}
$$

Therefore,

$$
\begin{aligned}
\dot{V}(\boldsymbol{z}) & \leq-2 \boldsymbol{z}^{*} C(\boldsymbol{z}) \boldsymbol{z}+\boldsymbol{z}^{*}\left[\mathcal{M}+\mathcal{M}^{*}\right] \boldsymbol{z} \\
& \leq-2 \sum_{i=1}^{N}\left|\boldsymbol{z}_{i}\right|^{4}+2 \bar{\mu} \sum_{i=1}^{N}\left|\boldsymbol{z}_{i}\right|^{2} \\
& \leq-2 \sum_{i=1}^{N}\left|\boldsymbol{z}_{i}\right|^{4}+2 \bar{\mu}|\boldsymbol{z}|^{2} .
\end{aligned}
$$

On the other hand, notice that

$$
\sum_{i=1}^{N}\left|\boldsymbol{z}_{i}\right|^{4} \geq \frac{1}{N}|\boldsymbol{z}|^{4}
$$

Indeed, we have

$$
|\boldsymbol{z}|^{4}=\left[\sum_{i=1}^{N}\left|z_{i}\right|^{2}\right]^{2}=\left[\sum_{i=1}^{N}\left|z_{i}\right|^{2}\right]\left|z_{1}\right|^{2}+\cdots+\left[\sum_{i=1}^{N}\left|z_{i}\right|^{2}\right]\left|z_{N}\right|^{2}
$$

so using the triangle inequality we see that, for each $j \leq N$,

$$
\left[\sum_{i=1}^{N}\left|z_{i}\right|^{2}\right]\left|z_{j}\right|^{2} \leq \frac{N}{2}\left|z_{j}\right|^{4}+\frac{1}{2} \sum_{i=1}^{N}\left|z_{i}\right|^{4}
$$

hence adding up the latter from $j=1$ to $N$, we obtain

$$
\left[\sum_{i=1}^{N}\left|z_{i}\right|^{2}\right]^{2} \leq N \sum_{j=1}^{N}\left|z_{j}\right|^{4}
$$


Therefore, substituting (42) in (41) we obtain

$$
\begin{aligned}
\dot{V}(\boldsymbol{z}) \leq & -\frac{2}{N}|\boldsymbol{z}|^{4}+2 \bar{\mu}|\boldsymbol{z}|^{2} \\
& =-\frac{1}{N}|\boldsymbol{z}|^{4}-\frac{1}{N}\left[|\boldsymbol{z}|^{2}-2 \bar{\mu} N\right]|\boldsymbol{z}|^{2} .
\end{aligned}
$$

Thus, from the last inequality, we conclude that $\dot{V}(\boldsymbol{z}) \leq-\frac{1}{N}|\boldsymbol{z}|^{4}$ for all $\boldsymbol{z}$ such that $|\boldsymbol{z}| \geq \sqrt{2 \bar{\mu} N}$. It follows, from Theorem (Khalil, 2002, Theorem 4.18), that the solutions are globally ultimately bounded and for any $R>0$ there exists a $T(R)$ such that for all initial conditions such that $\left|\boldsymbol{z}_{\circ}\right| \leq R$, the system's trajectories satisfy

$$
\left|\boldsymbol{z}\left(t, \boldsymbol{z}_{\circ}\right)\right| \leq \sqrt{2 \bar{\mu} N} \quad \forall t \geq T
$$

\subsection{Practical asymptotic stability of the synchronization errors manifold}

In this section we formulate conditions that ensure practical global asymptotic stability of the (not necessarily invariant) set

$$
\mathcal{S}=\left\{\boldsymbol{e} \in \mathbb{C}^{N}: e_{1}=e_{2}=\ldots=e_{N}=0\right\}
$$

We show that for large values of the interconnection gain $\gamma$ the norm of the error $\boldsymbol{e}(t)$ is small and inversely proportional to $\gamma$. More precisely, we establish that the set $\mathcal{S}$ is globally practically asymptotically stable for the system (39b). Our analysis relies on the following statement, which is reminiscent of results found in Corless and Leitmann (1981) for stability of an equilibrium.

Lemma 6.2. Consider the system $\dot{\boldsymbol{x}}=f(\boldsymbol{x})$, where $\boldsymbol{x} \in \mathbb{R}^{n}$ and $f: \mathbb{R}^{n} \rightarrow \mathbb{R}^{n}$ is continuous, locally Lipschitz. Assume that the system is forward complete and that there exist a closed set $\mathcal{A} \subset \mathbb{R}^{n}$, a $C^{1}$ function $V: \mathbb{R}^{n} \rightarrow \mathbb{R}_{+}$, functions $\alpha_{1}, \alpha_{2} \in \mathcal{K}_{\infty}$, $\alpha_{3} \in \mathcal{K}$ and a constant $c>0$ such that

$$
\begin{gathered}
\alpha_{1}\left(|\boldsymbol{x}|_{\mathcal{A}}\right) \leq V(\boldsymbol{x}) \leq \alpha_{2}\left(|\boldsymbol{x}|_{\mathcal{A}}\right) \\
\dot{V} \leq-\alpha_{3}\left(|\boldsymbol{x}|_{\mathcal{A}}\right)+c
\end{gathered}
$$

Then for any $R, \varepsilon>0$ there exists a $T=T(R, \varepsilon)$ such that for all $t \geq T$ and all $\boldsymbol{x}_{\circ} \in \mathbb{R}^{n}$ such that $\left|\boldsymbol{x}_{\circ}\right|_{\mathcal{A}} \leq R$

$$
\left|\boldsymbol{x}\left(t, \boldsymbol{x}_{\circ}\right)\right|_{\mathcal{A}} \leq r+\varepsilon,
$$

where $r=\alpha_{1}^{-1} \circ \alpha_{2} \circ \alpha_{3}^{-1}(c)$.

Our main statement in this section is the following.

Theorem 6.3. Consider the system (15), (16) and let Assumption 1 be satisfied. Let $\gamma^{*}$ be such that $\Re e\left[\lambda_{2}\left(A_{\gamma^{*}}\right)\right]<0$. Then, the set $\mathcal{S}$ is uniformly globally practically asymptotically stable for all $\gamma \geq \gamma^{*}$. Moreover, there exist $T^{*}>0, c_{1}, c_{2}>0$, inde- 
pendent of $\gamma$, such that synchronization errors $\boldsymbol{e}(t)$ satisfy

$$
|\boldsymbol{e}(t)|^{2} \leq \frac{c}{\left|\Re e\left[\lambda_{2}\left(A_{\gamma^{*}}\right)\right]\right|} \quad \forall t \geq T^{*}
$$

The previous statement relies mostly upon two properties of the networked system, namely, the negative definiteness of the second smallest eigenvalue of the Laplacian matrix $L$ and uniform boundedness of the trajectories of the network. For a network of the Stuart-Landau oscillators with coupling gain $\gamma$ it establishes that, for a given arbitrary large ball of initial conditions $B_{R}=\left\{\boldsymbol{z}_{\circ} \in \mathbb{C}^{N}:\left|\boldsymbol{z}_{\circ}\right| \leq R\right\}$ and an arbitrarily small constant $\delta>0$, we can always find constants $\gamma(R, \delta)$ and $T^{*}(R, \delta)$ such that the synchronization errors $\boldsymbol{e}\left(t, \boldsymbol{z}_{\circ}\right)$ satisfy

$$
\left|\boldsymbol{e}\left(t, \boldsymbol{z}_{\circ}\right)\right| \leq \delta \quad \text { for all } t \geq T^{*} .
$$

Proof of Theorem 6.3. Let $\boldsymbol{z}_{\circ} \in \mathbb{C}$ be initial conditions such that $\left|\boldsymbol{z}_{\circ}\right| \leq R$, where the constant $R>0$ is arbitrary. Let Assumption 1 generate a complex orthogonal matrix $V_{\gamma}$ and define

$$
e_{v}:=V_{\gamma}^{\top} \boldsymbol{e}
$$

From the latter, $(39 \mathrm{~b})$ and $D=V_{\gamma} \Lambda_{2} V_{\gamma}^{\top}$, we have

$$
\dot{\boldsymbol{e}}_{v}=V_{\gamma}^{\top} V_{\gamma} \Lambda_{2} V_{\gamma}^{\top} \boldsymbol{e}+\lambda_{1} \boldsymbol{e}_{v}-V_{\gamma}^{\top} P C(\boldsymbol{z}) \boldsymbol{z},
$$

which, in view of the orthogonality of $V_{\gamma}$, is equivalent to

$$
\dot{\boldsymbol{e}}_{v}=\Lambda \boldsymbol{e}_{v}-V_{\gamma}^{\top} P C(\boldsymbol{z}) \boldsymbol{z} .
$$

where $\Lambda$ is defined in (27). However, by construction, the first among the $N$ equations in (46) is redundant. Indeed, on one hand, we have $\boldsymbol{e}_{v}=V_{\gamma}^{\top} \boldsymbol{z}-V_{\gamma}^{\top} \vartheta_{\mathbf{r}_{1}} z_{m}$ so, using the identity $V_{\gamma}^{\top} \vartheta_{\mathbf{r}_{1}}=\left[\begin{array}{llll}1 & 0 & \cdots & 0\end{array}\right]^{\top}$, we obtain

$$
\boldsymbol{e}_{v}=\left(\begin{array}{c}
\vartheta_{\mathbf{r}_{1}}^{\top} \boldsymbol{z} \\
\vdots \\
\vartheta_{\mathbf{r}_{\mathrm{N}}}^{\top} \boldsymbol{z}
\end{array}\right)-\left(\begin{array}{c}
z_{m} \\
0 \\
\vdots \\
0
\end{array}\right)=:\left[\begin{array}{c}
0 \\
\tilde{\boldsymbol{e}}_{v}
\end{array}\right]
$$

On the other hand, the first element of $V_{\gamma}^{\top} P C(\boldsymbol{z}) \boldsymbol{z}$ equals to zero since the first row of $V_{\gamma}^{\top} P$ is entirely constituted of zeros. To see this, we observe that

$$
V_{\gamma}^{\top} P=\left[\begin{array}{c}
\vartheta_{\mathbf{r}_{1}}^{\top} \\
\widetilde{V}_{\gamma}^{\top}
\end{array}\right]\left[I-\vartheta_{\mathbf{r}_{1}} \vartheta_{\mathbf{r}_{1}}^{\top}\right]
$$

and recall that, by definition, $\vartheta_{\mathbf{r}_{1}}^{\top} \vartheta_{\mathbf{r}_{1}}=1$.

Then, let us consider the Lyapunov function candidate $V\left(\boldsymbol{e}_{v}\right)=\left|\boldsymbol{e}_{v}\right|^{2}=\left|\tilde{\boldsymbol{e}}_{v}\right|^{2}$ which is positive definite relative to the set $\mathcal{S}$. To see this, we refer to (47) and observe 
that $V\left(\boldsymbol{e}_{v}\right)$ is positive definite with respect to the set $\left\{\tilde{\boldsymbol{e}}_{v}=0\right\}$. Evaluating the total derivative of $V$ along the trajectories of (46), we obtain

$$
\begin{aligned}
\dot{V}\left(\boldsymbol{e}_{v}\right) & =\boldsymbol{e}_{v}^{*}\left(\Lambda \boldsymbol{e}_{v}-V_{\gamma}^{\top} P C(\boldsymbol{z}) \boldsymbol{z}\right)+\left(\boldsymbol{e}_{v}^{*} \Lambda^{*}-\boldsymbol{z}^{*} C(\boldsymbol{z}) P^{*} \bar{V}_{\gamma}\right) \boldsymbol{e}_{v} \\
& =\boldsymbol{e}_{v}^{*}\left[\Lambda+\Lambda^{*}\right] \boldsymbol{e}_{v}+g\left(\boldsymbol{e}_{v}, \boldsymbol{z}\right)
\end{aligned}
$$

where

$$
g\left(\boldsymbol{e}_{v}, \boldsymbol{z}\right)=-\boldsymbol{e}_{v}^{*} V_{\gamma}^{\top} P C(\boldsymbol{z}) \boldsymbol{z}-\boldsymbol{z}^{*} C(\boldsymbol{z}) P^{*} \bar{V}_{\gamma} \boldsymbol{e}_{v} .
$$

Now, since $\boldsymbol{e}_{v}=\left[0 \tilde{\boldsymbol{e}}_{v}^{\top}\right]^{\top}$ and the first element of $\boldsymbol{z}^{*} C(\boldsymbol{z}) P^{*} \bar{V}_{\gamma}$ equals to zero, we obtain, along the systems' trajectories $\boldsymbol{z}(t)$,

$$
\dot{V}\left(\boldsymbol{e}_{v}\right) \leq \tilde{\boldsymbol{e}}_{v}^{*} \Re e\left[\lambda_{2}\left(A_{\gamma^{*}}\right)\right] \tilde{\boldsymbol{e}}_{v}+g\left(\boldsymbol{e}_{v}, \boldsymbol{z}(t)\right)
$$

where we used the fact that $\Re e\left[\lambda_{2}\left(A_{\gamma}\right)\right] \geq \Re e\left[\lambda_{i}\left(A_{\gamma}\right)\right]$ for all $i>2$ and, by assumption, $0>\Re e\left[\lambda_{2}\left(A_{\gamma}\right)\right]$ for all $\gamma \geq \gamma^{*}$, that is, the eigenvalues of $D$ are non-positive.

Next, we observe that Proposition 6.1 implies that the solutions of (39a) are globally ultimately bounded hence, for any $R>0$ and any initial conditions such that $\left|\boldsymbol{z}_{\circ}\right| \leq R$ there exists a constant $T>0$ such that

$$
\left|\boldsymbol{z}\left(t, \boldsymbol{z}_{\circ}\right)\right| \leq \sqrt{2 \bar{\mu} N} \quad \forall t \geq T .
$$

In turn, it follows from (32) and (45), that $z_{m}(t)$ and the synchronization errors $\boldsymbol{e}(t)$, hence $\boldsymbol{e}_{v}(t)$, are also uniformly globally ultimately bounded. Moreover, the bound depends only on $\bar{\mu}$ and $N$. Furthermore, the eigenvalues and eigenvectors of $A_{\gamma}$ are uniformly bounded in $\gamma$ hence, there exists a constant $c>0$, which depends on $\bar{\mu}$ and $N$ only, such that

$$
\left|g\left(\boldsymbol{e}_{v}, \boldsymbol{z}(t)\right)\right| \leq c .
$$

From this and (48) it follows that

$$
\dot{V}\left(\boldsymbol{e}_{v}(t)\right) \leq-\left|\Re e\left[\lambda_{2}\left(A_{\gamma^{*}}\right)\right]\right|\left|\tilde{\boldsymbol{e}}_{v}(t)\right|^{2}+c .
$$

By direct integration and invoking the comparison theorem, it follows that there exists $T^{*}>0$ such that

$$
\left|\tilde{\boldsymbol{e}}_{v}(t)\right|^{2} \leq \frac{c}{\left|\Re e\left[\lambda_{2}\left(A_{\gamma^{*}}\right)\right]\right|} \quad \forall t \geq T^{*}
$$

so, from (45), (47) and the orthogonality of $V_{\gamma}$ we obtain (44). Global practical asymptotic stability of $\mathcal{S}$ follows from the fact that $\lim _{\gamma^{*} \rightarrow \infty} \Re e\left[\lambda_{2}\left(A_{\gamma^{*}}\right)\right]=-\infty$.

\subsection{Practical asymptotic stability of the invariant set of the averaged oscillator}

To complete our analysis, we consider the behavior of the solutions $z_{m}(t)$ of $(39 \mathrm{a})$. Notice that this equation may be regarded as that of a single Stuart-Landau oscillator 
with a perturbation, that is,

$$
\dot{z}_{m}=\left(\lambda_{1}-\alpha\left|z_{m}\right|^{2}\right) z_{m}+u,
$$

with $u=f_{m}\left(z_{m}, \boldsymbol{e}\right)$. This equation has exactly the form (1) (with additive input). Therefore, generally speaking, we may use stability theory for perturbed systems with respect to decomposable sets Angeli and Efimov (2013); Panteley, Loría, and El Ati (2015). Indeed, the origin is an invariant set, but so is the orbit $\left|z_{m}\right|=\sqrt{\lambda_{1 \mathrm{R}} / \alpha_{\mathrm{R}}}$, where $\alpha$ is defined in (34), which is determined by the complex parameters of the systems in the network, $\mu_{i}$. More precisely, the invariant is given by

$$
\mathcal{W}:=\left\{z \in \mathbb{C}:|z|=\sqrt{\frac{\lambda_{1 \mathrm{R}}}{\alpha_{\mathrm{R}}}}\right\} \cup\{z=0\} .
$$

Theorem 6.4. Consider the network of Stuart-Landau oscillators defined by Equations (15), (16) with an undirected interconnections-graph and the averaged oscillator of the network defined by (32a), whose dynamics is given by equation (49). Let Assumption 1 be satisfied. Then, the system (49) has the asymptotic gain property and moreover for any $\varepsilon>0$ there exists a gain $\gamma \geq \gamma^{*}$ such that

$$
\limsup _{t \rightarrow+\infty}\left|z_{m}\left(t, z_{\circ}\right)\right|_{\mathcal{W}} \leq \varepsilon
$$

Proof. Let $\gamma \geq \gamma^{*}$ and $R>0$ be arbitrary and consider the system (15), (16) with initial conditions $\boldsymbol{z}_{\circ} \in \mathbb{C}$ such that $\left|\boldsymbol{z}_{\circ}\right| \leq R$. From Proposition 6.1 it follows that the solutions of the system (15), (16) are ultimately bounded hence, there exists a $T=T(R)$ such that (40) holds for all $t \geq T$.

Now, let us consider the dynamics of the averaged oscillator, (49), given by

$$
\dot{z}_{m}=\left(\lambda_{1}-\alpha\left|z_{m}\right|^{2}\right) z_{m}+f_{m}\left(z_{m}, \boldsymbol{e}\right),
$$

where $f_{m}\left(z_{m}, \boldsymbol{e}\right)$ is defined in $(35 \mathrm{~b})$. From the latter, we see that $f_{m}\left(z_{m}, \cdot\right)$ is Lipschitz on compacts of $z_{m}$. Moreover, due to the ultimate boundedness of solutions, $z_{m}(t)$ is uniformly bounded; therefore, there exists a constant $c_{3}>0$ such that, for all $t \geq T$, we have

$$
\left|f_{m}\left(z_{m}(t), \boldsymbol{e}(t)\right)\right| \leq c_{3}|\boldsymbol{e}(t)|
$$

Thus, invoking Theorem 3 of Panteley, Loría, and El Ati (2015) with $u(t)=$ $f_{m}\left(z_{m}(t), \boldsymbol{e}(t)\right)$ and $t \geq T$, and using (6), we see that the solutions of Equation (49) satisfy the bound

$$
\limsup _{t \rightarrow+\infty}\left|z_{m}\left(t, \boldsymbol{z}_{\circ}\right)\right|_{\mathcal{W}} \leq \eta\left(|\boldsymbol{e}|_{\infty}\right)
$$

where

$$
|\boldsymbol{e}|_{\infty}=\sup _{t \geq 0}|\boldsymbol{e}(t)|
$$


Furthermore, from Theorem 6.3, there exist constants $T^{*}>T$ and $c>0$, independent of $\gamma$, such that for all $t \geq T^{*}$, the synchronization errors $\boldsymbol{e}(t)$ satisfy (44). It follows that

$$
\limsup _{t \rightarrow+\infty}\left|z_{m}\left(t, \boldsymbol{z}_{\circ}\right)\right|_{\mathcal{W}} \leq \eta\left(\left[\frac{c}{\left|\Re e\left[\lambda_{2}\left(A_{\gamma^{*}}\right)\right]\right|}\right]^{1 / 2}\right) \quad \forall t \geq T^{*}
$$

and, by repeating the same argument as before, i.e., observing that

$$
\lim _{\gamma^{*} \rightarrow \infty} \Re e\left[\lambda_{2}\left(A_{\gamma^{*}}\right)\right]=-\infty,
$$

and using the fact that $\eta \in \mathcal{K}_{\infty}$, we obtain that, for any $\varepsilon>0$, there exists a $\gamma>\gamma^{*}$ such that

$$
\eta\left(\left[\frac{c}{\left|\Re e\left[\lambda_{2}\left(A_{\gamma^{*}}\right)\right]\right|}\right]^{1 / 2}\right) \leq \varepsilon
$$

We conclude that

$$
\limsup _{t \rightarrow+\infty}\left|z_{m}\left(t, \boldsymbol{z}_{\circ}\right)\right|_{\mathcal{W}} \leq \varepsilon .
$$

That is, the invariant set $\mathcal{W}$ is practically asymptotically stable, in the sense that, by increasing the interconnection gain $\gamma$, we can make solutions $z_{m}\left(t, \boldsymbol{z}_{\circ}\right)$ converge arbitrarily close to $\mathcal{W}$.

\section{Simulations}

To illustrate our theoretical findings, we have performed some numerical simulations, based on the case-study presented in Bergner et al. (2012). We consider a group of four Stuart-Landau oscillators with natural frequencies $\omega_{i} \in\{2.5,0.975,0.992,1.008\}$, unitary amplitude, and interconnected in a star topololgy defined by the following Laplacian

$$
L=\left[\begin{array}{cccc}
3 & -1 & -1 & -1 \\
-1 & 1 & 0 & 0 \\
-1 & 0 & 1 & 0 \\
-1 & 0 & 0 & 1
\end{array}\right]
$$

We performed several numerical simulations for different values of the interconnection gain $\gamma$. In Figure 2 are showed some of these results in terms of the trajecories of $z_{i \mathrm{R}}(t)$; one may appreciate the synchronized behaviour of the oscillators as $\gamma$ is increased.

Furthermore, we have proceeded to compute the synchronization frequency for each value of the interconnection gain using both, the numirecal data obtained from the simulations and the analyitycal formula (5) applied to the emergent-dynamics oscillator (36). In the latter $\lambda_{1}$ corresponds to the eigen-value of $A_{\gamma}$, as defined in (17b), with the largest real part and $\alpha$ is defined in (34). In Figure 3 we show the splineinterpolated values of the estimated synchronization frequencies, in function of $\gamma$. It 

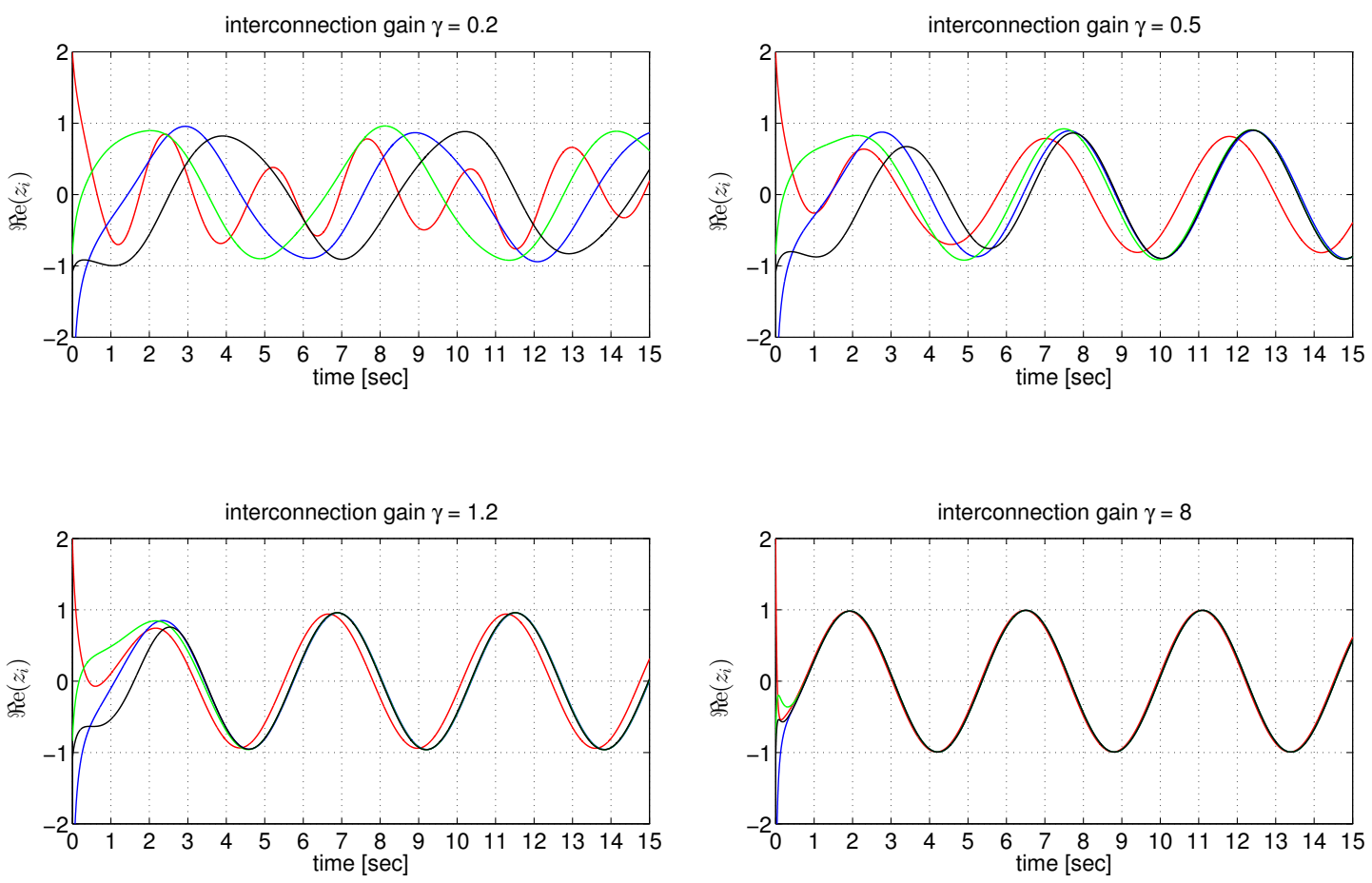

Figure 2. Systems' responses in terms of $z_{i \mathrm{R}}(t)$ for different values of the interconnection gain $\gamma$

may be appreciated that this frequency is in the neighbourhood of $1.3685 \mathrm{rad} / \mathrm{s}$ for large values of the interconnection strength.

\section{Conclusions}

We have established that a network of heterogeneous nonlinear oscillators achieve practical dynamic consensus under diffusive coupling. The synchronization errors are ultimately bounded and may be diminished by enlarging the coupling strength. These are, however, preliminary analysis results. Important control design problems are completely open. For instance, under which circumstances, one may control the emergent dynamics and, hence, the collective behavior of the networked systems. Furthermore,

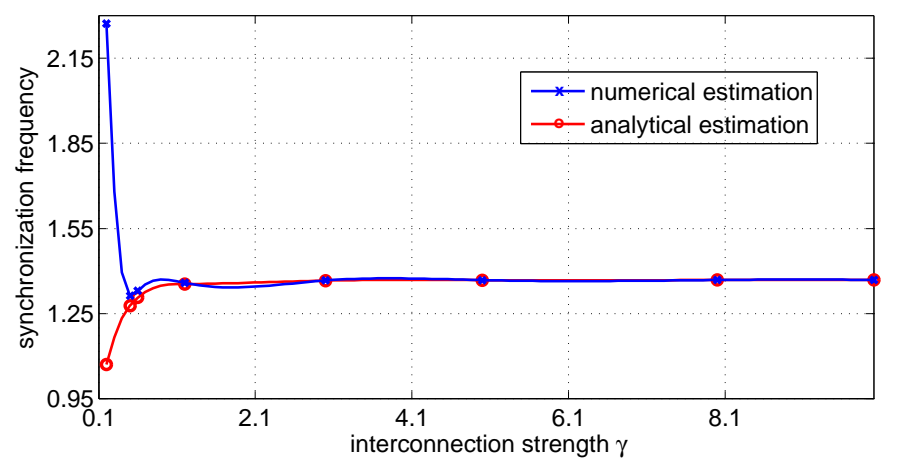

Figure 3. Comparison of the synchronization frequencies for different values of the interconnection gain based on numerical simulations and the analytical value 
we established dynamic consensus only in a practical sense, that is, we proved that the systems' trajectories remain within a neighbourhood of the emergent dynamics attractor. Establishing asymptotic dynamic consensus for heterogeneous systems that is, that all the systems' trajectories tend asymptotically to the attractor, is a challenging problem under investigation.

\section{References}

Andronov, A. A., Vitt, A. A., \& Khakin, S. E. (1987). Theory of oscillators. Dover Mathematics. (Original in Russian, 1959.)

Angeli, D., \& Efimov, D. (2013). On input-to-state stability with respect to decomposable invariant sets. In Proc. of the 52nd ieee conference on decision and control (pp. 5897-5902). Florence, Italy.

Aoyagi, T. (1995). Network of neural oscillators for retrieving phase information. Phys. Rev. Lett., 74, 4075-4078.

Belhaq, M., \& Houssni, M. (2000). Suppression of chaos in averaged oscillator driven by external and parametric excitations. Chaos, Solitons and Fractals, 11(8), 1237-1246.

Bergner, A., Frasca, M., Sciuto, G., Buscarino, A., Ngamga, E. J., Fortuna, L., \& Kurths, J. (2012, Feb). Remote synchronization in star networks. Phys. Rev. E, 85, 026208. Retrieved from https://link.aps.org/doi/10.1103/PhysRevE. 85.026208

Cagnan, H., Meijer, H. G., Gils, S. A., Krupa, M., Heida, T., Rudolph, M., ... Martens, H. C. (2009). Frequency-selectivity of a thalamocortical relay neuron during Parkinson's disease and deep brain stimulation : a computational study. European Journal of Neuroscience, $30(7), 1306-1317$.

Carr, T., Taylor, M., \& Schwartz, I. (2006). Negative-coupling resonances in pump-coupled lasers. Physica D: Nonlinear Phenomena, 213(2), 152 - 163.

Corless, M., \& Leitmann, G. (1981). Continuous state feedback guaranteeing uniform ultimate boundedness for uncertain dynamic systems. IEEE Trans. on Automatic Control, 26(5), 1139-1144.

Craven, B. D. (1969). Complex symmetric matrices. Journal of the Australian Mathematical Society, 10, 341-354.

Dunn, J. M., \& Anderssen, R. S. (2011). A review of models used for understanding epileptic seizures. In International Congress on Modelling and Simulation. Perth.

Franci, A., Chaillet, A., Panteley, E., \& Lamnabhi-Lagarrigue, F. (2012). Desynchronization and inhibition of Kuramoto oscillators by scalar mean-field feedback. Mathematics of Control, Signals and Systems, 24(1-2), 169-217.

Franci, A., Scardovi, L., \& Chaillet, A. (2011). An input-output approach to the robust synchronization of dynamical systems with an application to the Hindmarsh-Rose neuronal model. In Proc. of the joint ieee conference on decision and control and european control conference (p. 6504-6509).

Horn, R. A., \& Johnson, C. R. (1985). Matrix analysis. Cambridge Press.

Ipsen, M., Hynne, F., \& Soerensen, P. G. (1997, November). Amplitude equations and chemical reaction-diffusion systems.

Jouffroy, J., \& Slotine, J. J. (2004). Methodological remarks on contraction theory. In Proc. of the 43rd ieee conference on decision and control (Vol. 3, p. 2537-2543).

Karnatak, R., Ramaswamy, R., \& Prasad, A. (2007). Amplitude death in the absence of time delays in identical coupled oscillators. Phys. Rev. E, 76, 035201.

Khalil, H. (2002). Nonlinear systems. New York: Prentice Hall, 3rd ed.

Kuramoto, Y. (1975). Self-entrainment of a population of coupled non-linear oscillators. Lecture Notes in Physics, 39, 420-422.

Kuznetsov, Y. A. (1998). Elements of applied bifurcation theory. Springer, Applied Mathematical Sciences, Vol. 112. 
Lehnert, J., Hövel, P., Selivanov, A., Fradkov, A. L., \& Schöll, E. (2014). Controlling cluster synchronization by adapting the topology. Physical Review E, $90(4), 042914$.

Lohmiller, W., \& Slotine, J. J. (2005). Contraction analysis of non-linear distributed systems. International Journal of Control, 78, 678-688.

Mallet, N., Pogosyan, A., Marton, F., Bolam, J. P., Brown, P., \& Magill, P. J. (2008). Parkinsonian Beta oscillations in the External Globus Pallidus and their relationship with Subthalamic Nucleus activity. Journal of Neuroscience, 28(7), 14245-14258.

Matthews, P. C., Mirollo, R. E., \& Strogatz, S. H. (1991). Dynamics of a large system of coupled nonlinear oscillators. Physica D: Nonlinear Phenomena, 52, 293 - 331.

Mormann, F., Lehnertz, K., David, P., \& Elger, C. E. (2000). Mean phase coherence as a measure for phase synchronization and its application to the eeg of epilepsy patients. Physica D: Nonlinear Phenomena, 144(34), 358 - 369.

Moro, J., Burke, J., \& Overton, M. (1997). On the Lidskii-Vishik-Lyusternik perturbation theory for eigenvalues of matrices with arbitrary Jordan structure. SIAM Journal on Matrix Analysis and Applications, 18(4), 793-817.

Olfati-Saber, R., \& Murray, R. (2004). Consensus problems in networks of agents with switching topology and time-delays. Automatic Control, IEEE Transactions on, 49(9), 1520 1533.

Panteley, E., \& Loria, A. (2017). Synchronization and dynamic consensus of heterogeneous networked systems. IEEE Trans. Automat. Control, 62(8), 3758-3773. (Pre-published online. DOI: 10.1109/TAC.2017.2649382)

Panteley, E., Loría, A., \& El Ati, A. (2015). Analysis and control of andronov-hopf oscillators with applications to neuronal populations. In Proc. 54th IEEE Conf. Decision and Control (pp. 596 - 601). Osaka, Japan. (DOI: 10.1109/CDC.2015.7402294)

Panteley, E., Loría, A., \& El Ati, A. (2015). On the stability and robustness of StuartLandau oscillators. in Proc. 1st IFAC Conference onModelling, Identification andControl of Nonlinear Systems, MICNON 2015, (St. Petersburg, Russia). IFAC-PapersOnLine, 48(11), 645 - 650. (DOI: 10.1016/j.ifacol.2015.09.260)

Perko, L. (2000). Differential equations and dynamical systems. Springer.

Pham, Q. C., \& Slotine, J. J. (2007). Stable concurrent synchronization in dynamic system networks. Neural Networks, 20(1), 62-77.

Pogromsky, A. Y., Glad, T., \& Nijmeijer, H. (1999). On difffusion driven oscillations in coupled dynamical systems. International Journal of Bifurcation and Chaos in Applied Sciences and Engineering, 9(4), 629-644.

Pogromsky, A. Y., \& Nijmeijer, H. (2001). Cooperative oscillatory behavior of mutually coupled dynamical systems. IEEE Trans. on Circuits and Systems I: Fundamental Theory and Applications, 48(2), 152-162.

Qin, Y.-M., Men, C., Zhao, J., Han, C.-X., \& Che, Y.-Q. (2018). Toward heterogeneity in feedforward network with synaptic delays based on fitzhughnagumo model. International Journal of Modern Physics B, 32(01), 1750274. Retrieved from https://www . worldscientific.com/doi/abs/10.1142/S0217979217502745

Ren, W., Beard, R., \& Atkins, E. (2007, April). Information consensus in multivehicle cooperative control. Control Systems, IEEE, $27(2), 71-82$.

Rosenblum, M. G., \& Pikovsky, A. S. (2004). Controlling synchronization in an ensemble of globally coupled oscillators. Physical Review Letters, 92(11).

Sakaguchi, H., \& Kuramoto, Y. (1986). A soluble active rotater model showing phase transitions via mutual entertainment. Progress of Theoretical Physics, 76 (3), 576-581.

Scardovi, L., Arcak, M., \& Sontag, E. D. (2009). Synchronization of interconnected systems with an input-output approach. Part I: Main results. In Proc. of the 48 th ieee conference on decision and control (p. 609-614).

Selivanov, A., Lehnert, J., Dahms, T., Hövel, P., Fradkov, A. L., \& Schöll, E. (2012, 01). Adaptive synchronization in delay-coupled networks of stuart-landau oscillators. Physical Review E, 85, 016201.

Steur, E., Tyukin, I., Gorban, A., Jarman, N., Nijmeijer, H., \& van Leeuwen, 
C. (2016). Coupling-modulated multi-stability and coherent dynamics in directed networks of heterogeneous nonlinear oscillators with modular topology. IFAC-PapersOnLine, $49(14), 62$ - $67 . \quad$ Retrieved from http://www.sciencedirect.com/science/article/pii/S2405896316312691 (6th IFAC Workshop on Periodic Control Systems PSYCO 2016)

Strogatz, S. H. (2000). From kuramoto to crawford: exploring the onset of synchronization in populations of coupled oscillators. Physica D: Nonlinear Phenomena, 143(1-4), 1 - 20.

Sune, D., F. Madsen, M., \& G. Sorensen, P. (2005). Chemical interpretation of oscillatory modes at a hopf point. Phys. Chem. Chem. Phys., 7, 1674-1679.

Teel, A. R., Peuteman, J., \& Aeyels, D. (1999). Semi-global practical asymptotic stability and averaging. Systems and Control Letters, 37(5), 329-334.

Teramae, J. N., \& Tanaka, D. (2004). Robustness of the noise-induced phase synchronization in a general class of limit cycle oscillators. Phys. Rev. Lett., 93, 204103.

Wang, L., Chen, M. Z., \& Wang, Q.-G. (2015). Bounded synchronization of a heterogeneous complex switched network. Automatica, 56, 19 - 24. Retrieved from http://www.sciencedirect.com/science/article/pii/S0005109815001314

Wilkinson, J. H. (1965). The algebraic eigenvalue problem (Vol. 87). Clarendon Press Oxford.

Zhang, F., Trentelman, H. L., \& Scherpen., J. M. A. (2016). Robust cooperative output regulation of heterogeneous lur'e networks. International Journal of Robust and Nonlinear Control, 27(16), 3061-3078. Retrieved from https://onlinelibrary.wiley.com/doi/abs/10.1002/rnc. 3725 